\title{
Asymptotic Solutions for Optical Properties of Large Particles with
}

\section{Strong Absorption}

Ping Yang ${ }^{1,4}$, Bo-Cai $\mathrm{Gao}^{2}$, Bryan A. Baum ${ }^{3}$, Yong X. $\mathrm{Hu}^{3}$, Warren J. Wiscombe, Michael I. Mishchenko ${ }^{5}$, Dave M. Winker ${ }^{3}$, Shaima L. Nasiri ${ }^{6}$

1. Scientific Systems and Applications Inc. Lanham, MD

2. Remote Sensing Division, Naval Research Laboratory, Washington, D.C.

3. NASA Langley Research Center, Hampton, VA

4. NASA Goddard Space Fight Study Center, Greenbelt, MD

5. NASA Goddard Institute for Space Studies, New York

6. Cooperative Institute for Meteorological Satellite Studies/University of Wisconsin, Madison, WI

For publication in

Applied Optics

OCIS codes: $010.1290,010.1310,010.3920,290.5850,290.1090,280.1100$

Corresponding author address: Dr. Ping Yang, Code 913, NASA/Goddard Space Flight Study Center, Greenbelt, MD 20771; Tel: 301-614-6127; Fax: 301-614-6307; email: pyang@climate.gsfc.nasa.gov. 


\begin{abstract}
For scattering calculations involving nonspherical particles such as ice crystals, we show that the transverse wave condition is not applicable to the refracted electromagnetic wave in the context of geometric optics when absorption is involved. Either the TM wave condition (i.e., where the magnetic field of the refracted wave is transverse with respect to the wave direction) or the TE wave condition (i.e., where the electric field is transverse with respect to the propagating direction of the wave) may be assumed for the refracted wave in an absorbing medium to locally satisfy the electromagnetic boundary condition in the ray tracing calculation. The wave mode assumed for the refracted wave affects both the reflection and refraction coefficients. As a result, a nonunique solution for these coefficients is derived from the electromagnetic boundary condition. In this study we have identified the appropriate solution for the Fresnel reflection/refraction coefficients in light scattering calculation based on the ray tracing technique. We present the $3 \times 2$ refraction or transmission matrix that completely accounts for the inhomogeneity of the refracted wave in an absorbing medium. Using the Fresnel coefficients for an absorbing medium, we derive an asymptotic solution in an analytical format for the scattering properties of a general polyhedral particle. Numerical results are presented for hexagonal plates and columns with both preferred and random orientations. The asymptotic theory can produce reasonable accuracy in the phase function calculations in the infrared window region (wavelengths near $10 \mu \mathrm{m}$ ) if the particle size (in diameter) is on the order of $40 \mu \mathrm{m}$ or larger. However, since strong absorption is assumed in the computation of the single-scattering albedo in the asymptotic theory, the single scattering albedo does not change with variation of the
\end{abstract}


particle size. As a result, the asymptotic theory can lead to substantial errors in the computation of single-scattering albedo for small and moderate particle sizes. However, from comparison of the asymptotic results with the FDTD solution, it is expected that a convergence between the FDTD results and the asymptotic theory results can be reached when the particle size approaches $200 \mu \mathrm{m}$. We show that the phase function at sidescattering and backscattering angles is insensitive to particle shape if the random orientation condition is assumed. However, if preferred orientations are assumed for particles, the phase function has a strong dependence on scattering azimuthal angle. The single-scattering albedo also shows very strong dependence on the inclination angle of incident radiation with respect to the rotating axis for the preferred particle orientations. 


\section{Introduction}

The optical properties of dielectric particles such as aerosols and ice crystals in the atmosphere are fundamental to a number of disciplines including atmospheric radiation transfer and air-borne or satellite-borne remote sensing applications. In a recent book $^{1}$, Mishchenko et al. have comprehensively reviewed various methods that have been developed to solve the scattering and absorption properties of particles for a variety of geometric morphologies and electromagnetic characteristics of the scatterers. Accurate

numerical methods such as the discrete dipole approximation (DDA) method ${ }^{2.3}$ and the finite-difference time domain (FDTD) technique ${ }^{4,5}$ are applicable in practice only to size parameters smaller than 20 because the computational requirements increase quickly with size parameter. Although analytical solutions are available for some particle shapes such as spheres ${ }^{6}$ and spheroids ${ }^{7,8}$, the corresponding numerical computations are usually very challenging. For example, the computation of the optical properties of spheroids based on an analytical solution in terms of a series of special functions may not be stable when the size parameter is larger than approximately $30-40$. It is worth noting that the recent developments on the T-matrix method ${ }^{9,10}$ allow the exact solution for the optical properties of spheroids and finite circular cylinders for size parameters up to $200^{11}$. There is no single method to solve for the optical properties of nonspherical particles across the entire size parameter spectrum.

It is common to use the ray-tracing technique ${ }^{12-15}$ for nonspherical particles that have sizes much larger than the incident wavelength. Previous applications of the raytracing technique have been focused mainly on scattering computations for the visible and near-infrared wavelengths for which the imaginary part of the refractive index is 
small. For these weakly absorptive spectra, the conventional Snell's law and Fresnel formulae can be used to account for the ray reflection and refraction at particle surface. However, the optical properties of large dielectric particles at wavelengths with strong absorption are fundamental to many practical applications. For example, the scattering and absorption properties of ice crystals at infrared wavelengths are required in the retrieval of cirrus clouds using satellite radiance data such as that from the Advanced Very High Resolution Radiometer (AVHRR) ${ }^{16}$ and Moderate Resolution Imaging Spectroradiometer (MODIS) ${ }^{17}$ imagers. Under cirrus cloudy condition, interpretation of the High resolution Interferometer Sounder (HIS) ${ }^{18}$ hyperspectrum in the atmospheric window region $(8-12 \mu \mathrm{m})$ also requires the optical properties of ice crystals.

When ray optics is applied to the infrared spectrum in regions of strong absorption, the refracted wave inside the particle is inhomogeneous in the sense that the planes of constant phase and constant amplitude are not parallel ${ }^{19}$. Current applications of the ray tracing technique assume that planes of constant phase and constant amplitude are parallel. The inhomogeneity associated with the reflected wave substantially complicates the behaviors of rays at particle surface and inside the medium. Stratton ${ }^{19}$ and Bom and Wolf ${ }^{0}$ have discussed this inhomogeneity and its effect on the Fresnel reflection coefficients associated with complex refractive index. In particular, Stratton ${ }^{19}$ (section 9.9) has solved the problem of the reflection of a plane electromagnetic wave by a conducting surface. Recently, Zhang and $\mathrm{Xu}^{21}$ and Yang and Liou ${ }^{22}$ addressed the effect of the inhomogeneous wave on ray-tracing calculation. Zhang and $\mathrm{Xu}^{21}$ have expressed the Fresnel coefficients without an explicit use of complex refractive index. However, they did not account for the effect of the inhomogeneity on wave attenuation that is the 
most important physical process involved in the ray propagation within an absorbing medium. Yang and Liou $^{22}$ have discussed the impact of the inhomogeneous effect on ray propagation by introducing an effective refractive index, but they employed the highfrequency approximation ${ }^{20}$ in the calculation of reflection and refraction coefficients. Since the effect of inhomogeneity on ray tracing calculation has not been solved completely, it is necessary to further study this problem. The intent of this study is to develop an analytical asymptotic method that comprehensively accounts for the inhomogeneity effect when deriving the optical properties (such as the single-scattering albedo and phase function) of strongly absorbing particles with sizes much greater than the incident wavelength.

To compute the optical properties of dielectric particles with strong absorption we first derive the reflection and refraction coefficients for the electric field in terms of an apparent refractive index without using the high-frequency approximation. The reflection and refraction coefficients incorporate the inhomogeneous effect on the direction of ray propagation and the Fresnel coefficients. Subsequently, we derive the asymptotic solution expressed in an analytical form for large particles with strong absorption. In Section 2 we discuss the various wave modes of an inhomogeneous wave refracted into a particle when absorption is involved. We show that the electric or magnetic field may not be perpendicular to the direction of ray propagation because of the effect of strong absorption. The Fresnel coefficients are reformulated, in particular, the transmission coefficient for the component of the refracted field that is parallel to ray propagation. In Section 3, we present the analytical solution for the optical properties of large polyhedral particles with strong absorption. The solution is derived on the basis of the 
electromagnetic relationship between the near field and far field. In Section 3, we also present numerical results of the asymptotic solution for hexagonal ice crystals. Finally, conclusions of this study are given in Section 4.

\section{Physical Basis for Ray Tracing in Absorptive Case}

In this section, it is shown that the transverse wave condition cannot be applied to both the electric and magnetic fields associated with a ray refracted into an absorbing medium. In addition, we show the wave mode subject to an electric boundary condition is not unique in the context of geometric optics when absorption is involved. The behavior of the refracted wave not only affects the Fresnel refraction coefficients but also the Fresnel reflection coefficients. In the following discussion, it is shown that there are four combinations possible for the reflection/refraction coefficients that depend on wave modes. While each combination of the reflection and refraction coefficients is a mathematically rational solution, only one set of coefficients should be used in the ray tracing solution. The choice of the proper wave mode for the refracted wave in ray tracing calculation is made through the comparison of the geometric optics solution to the analytical Mie result for the scattering properties of a very large (size parameter on the order of 1000) sphere with strong absorption.

Let us consider the application of the geometric optics method to solve for the scattering properties of a particle that is large and absorptive. In this case, the rays that are refracted into the particle are largely absorbed. Thus, we need only consider the first order reflected/refracted rays because of the small amount of energy carried by the rays that undergo internal reflections. To examine the first order reflection/refraction 
characteristics and the corresponding polarization features of the electric field, we begin with the phase variation for the field associated with a complex refractive index. Let three unit vectors, $\hat{e}_{i}, \hat{e}_{r}$, and $\hat{e}_{t}$, indicate the propagating directions for the incident, reflected, and refracted rays. To systematically present this study, it is necessary to recapitulate some results obtained by Yang and $\mathrm{Liou}^{22}$. Following these authors, the fields associated with these rays can be written as follows:

$$
\begin{aligned}
& \vec{E}_{i}(\vec{r})=\bar{E}_{i o} \exp \left(i k \hat{e}_{i} \cdot \vec{r}\right), \\
& \vec{E}_{r}(\vec{r})=\vec{E}_{r o} \exp \left(i k \hat{e}_{r} \cdot \vec{r}\right), \\
& \vec{E}_{t}(\vec{r})=\vec{E}_{t o} \exp \left[i k\left(N_{r} \hat{e}_{t}+i N_{n} \hat{n}\right) \cdot \vec{r}\right],
\end{aligned}
$$

where $\vec{E}_{i, o}, \vec{E}_{r, o}$, and $\bar{E}_{t, o}$ are the amplitude vectors for the fields; $k=2 \pi / \lambda$ in which $\lambda$ is the wavelength in vacuum; $\hat{n}$ is a unit vector that is normal to particle surface and points into the particle. The two parameters $N_{r}$ and $N_{n}$ in Eq.(1c) are given by

$$
\begin{aligned}
& N_{r}=2^{-1 / 2}\left\{m_{r}^{2}-m_{i}^{2}+\sin ^{2} \zeta_{i}+\left[\left(m_{r}^{2}-m_{i}^{2}-\sin ^{2} \zeta_{i}\right)^{2}+4 m_{r}^{2} m_{i}^{2}\right]^{1 / 2}\right\}, \text { and } \\
& N_{n}=2^{-1 / 2}\left\{-\left(m_{r}^{2}-m_{i}^{2}-\sin ^{2} \zeta_{i}\right)+\left[\left(m_{r}^{2}-m_{i}^{2}-\sin ^{2} \zeta_{i}\right)^{2}+4 m_{r}^{2} m_{i}^{2}\right]^{1 / 2}\right\}
\end{aligned}
$$

in which $m_{r}$ and $m_{i}$ are the real and imaginary part of the refractive index, and $\zeta_{i}$ is the incident angle given by $\zeta_{i}=\sin ^{-1}\left[1-\left(\hat{e}_{i} \cdot \hat{n}\right)^{2}\right]^{1 / 2}$. Note that $N_{n}$ (denoted as $N_{i}^{*}$ in Ref. 22) is not presented in the previous study. Physically, $N_{r}$ is the parameter that determines the phase and $N_{n}$ is the parameter that determines the absorption. This will be illustrated in the following discussion.

From Eqs.(1a)-(1c) and the continuity of wave phase at the medium interface, it follows that Snell's law for an absorbing medium can be mathematically expressed in the form of 


$$
\begin{aligned}
& \hat{e}_{r}=\hat{e}_{i}+2\left(\hat{e}_{i} \cdot \hat{n}\right) \hat{n}, \\
& \hat{e}_{t}=\hat{e}_{i} / N_{r}+\left(\cos \zeta_{t}-\cos \zeta_{i} / N_{r}\right) \hat{n},
\end{aligned}
$$

where the refractive angle is given by $\zeta_{t}=\sin ^{-1}\left[\sin \left(\zeta_{i}\right) / N_{r}\right]$. Equation (2a) means that the incident and reflected rays are spatially symmetric about the direction normal to particle surface at the incident point, which is the standard Snell's law for reflection direction. However, Eq. (2b) indicates that the refracted direction is not determined by $m_{r}$ but by $N_{r}$. For this reason, $N_{r}$ is referred to as the real part of apparent refractive index, which depends on the incident configuration and the dielectric constant of the medium for the determination of the direction and phase for refracted wave. The attenuation of the refracted field due to the absorption depends on the path of observation. If the path is along $\hat{n}$, the attenuation is determined by $N_{n}$, as is evident from Eq.(1c). However, if we trace the refracted wave along the direction of the refracted ray, i.e., along position vector $\vec{r}=l \hat{e}_{t}$ in which $l$ is the penetration depth of the ray, the electric field is given by

$$
\vec{E}_{t}\left(l \hat{e}_{t}\right)=\bar{E}_{t o} \exp \left[-k N_{n}\left(\hat{n} \cdot \hat{e}_{t}\right) l\right] \exp \left(i k N_{r} l\right) .
$$

Obviously, the factor $N_{n}\left(\hat{n} \cdot \hat{e}_{t}\right)$ is the imaginary part of the apparent refractive index that governs the attenuation of the electric field associated with the rays. We denote this factor as $N_{i}$. Thus, for a ray impinging on an absorbing medium, the apparent refractive index in the complex format is $\left(N_{r}+i N_{i}\right)$. It can be proven that

$$
N_{i}=N_{n}\left(\hat{n} \cdot \hat{e}_{t}\right)=N_{n} \cos \zeta_{t}=m_{r} m_{i} / N_{r} .
$$

Figure 1 shows the real and imaginary parts of the inherent and apparent refractive index of ice at infrared wavelengths 11 and $12 \mu \mathrm{m}$ for which substantial absorption exists. The optical properties of ice at these two wavelengths are important 
for many applications in satellite remote sensing ${ }^{17}$. Note that for ice particles, wavelengths near $11 \mu \mathrm{m}$ are in the Christiansen band where absorption dominates the extinction ${ }^{23}$. The inherent refractive index is determined uniquely by the dielectric characteristics of the medium, i.e., permittivity, which does not depend on the incident configuration of a ray. As is shown in Fig.1, the real part of the apparent refractive index increases with the increase of incident angle whereas the imaginary part of the refractive index decreases with the increase of the incident angle. Obviously, in terms of wave attenuation per unit length along propagating direction of a ray, the particle is less absorptive at a large incident angle. For incident angles larger than $10^{\circ}$, the inhomogeneous effect due to strong absorption cannot be ignored.

It can be shown that various wave modes may satisfy the electromagnetic boundary condition associated with the reflection and refraction at the interface of two media, one of which is strongly absorptive. First, let $\hat{\beta}$ be a unit vector perpendicular to the incident plane. The unit vectors that are parallel to the incident plane and perpendicular to ray propagation directions for the incident, reflected, and refracted waves are given by

$$
\hat{\alpha}_{i}=\hat{e}_{i} \times \hat{\beta}, \quad \hat{\alpha}_{r}=\hat{e}_{r} \times \hat{\beta} \text {, and } \hat{\alpha}_{t}=\hat{e}_{t} \times \hat{\beta}
$$

It should be pointed out that $\hat{\beta} \hat{\alpha}_{i, r, t} \hat{e}_{i, r, t}$ constitute right-handed coordinate systems. The incident and reflected waves are within non-absorbing medium. As a result of the homogeneous wave properties of the incident and reflected rays, transverse wave condition can be imposed on both electric and magnetic fields for these rays (hereafter, referred to as TEM mode). Thus, the corresponding amplitude vectors of the electric fields can be decomposed in the form of 


$$
\begin{aligned}
& \vec{E}_{i o}=E_{i o, \alpha} \hat{\alpha}_{i}+E_{i o, \beta} \hat{\beta}, \\
& \vec{E}_{r o}=E_{r o, \alpha} \hat{\alpha}_{r}+E_{r o, \beta} \hat{\beta} .
\end{aligned}
$$

The Maxwell equations in time-independent form are given by

$$
\begin{aligned}
& \bar{E}(\vec{r})=\nabla \times \bar{H}(\vec{r}) /(-i k \varepsilon), \\
& \bar{H}(\vec{r})=\nabla \times \bar{E}(\bar{r}) / i k,
\end{aligned}
$$

where $\varepsilon$ is permittivity given by $\varepsilon=\left(m_{r}+i m_{i}\right)^{2}$. We have chosen the time harmonic factor as $\exp (i k c t)$ in which $\mathrm{c}$ is the speed of light in vacuum. If this factor is selected as $\exp (-i k c t)$, the sign of the imaginary part of refractive index is negative $e^{1,24}$. Using Eq.(7b), we can obtain the amplitude vectors of magnetic fields associated with the incident and reflected rays as follows:

$$
\begin{aligned}
& \bar{H}_{i o}=\hat{e}_{i} \times\left(E_{i o, \alpha} \hat{\alpha}_{i}+E_{i o, \beta} \hat{\beta}\right)=E_{i o, \beta} \hat{\alpha}_{i}-E_{i o, \alpha} \hat{\beta}, \\
& \bar{H}_{r o}=\hat{e}_{r} \times\left(E_{r o, \alpha} \hat{\alpha}_{r}+E_{r o, \beta} \hat{\beta}\right)=E_{r o, \beta} \hat{\alpha}_{r}-E_{r o, \alpha} \hat{\beta} .
\end{aligned}
$$

For the refractive wave in an absorbing medium, a transverse wave condition on both the electric and magnetic fields cannot be imposed because of the inhomogeneity effect, that is, the TEM wave mode is not valid in this case.

In the following discussion, we first examine the reflecting characteristics of an absorbing medium for various wave modes. To derive the reflection coefficient, the electric field may be assumed to be transverse with respect to the propagating direction of the ray (hereafter, this mode is referred to as TE mode) so that the refracted electric field can be expressed in the form of

$$
\bar{E}_{t o}=E_{t o, \alpha} \hat{\alpha}_{t}+E_{t o, \beta} \hat{\beta}
$$


From Eqs. (1c), (4), (7b), and (9) and the relationship of $\hat{n}=\cos \zeta_{t} \hat{e}_{t}-\sin \zeta_{t} \hat{\alpha}_{t}$, the refracted magnetic field in TE mode can be obtained as follows:

$$
\begin{aligned}
& \vec{H}_{t o}(\vec{r})=\left(N_{r} \hat{e}_{t}+i N_{n} \hat{n}\right) \times\left(E_{t o, \alpha} \hat{\alpha}_{t}+E_{t o, \beta} \hat{\beta}\right) \\
& =\left(N_{r}+i N_{i}\right) E_{t o, \beta} \hat{\alpha}_{t}-\left(N_{r}+i N_{i}\right) E_{t o, \alpha} \hat{\beta}+i N_{n} \sin \zeta_{t} E_{t o, \beta} \hat{e}_{t} .
\end{aligned}
$$

From Eq.(10), it is evident that the refracted magnetic field has a nonzero component along the unit vector $\hat{e}_{t}$ (ray direction) when $N_{n}$ is not zero, i.e., when absorption is involved. At the interface of two media, the electromagnetic boundary condition requires that the tangential components of electric and magnetic fields are continuous. Thus, we have the following relationships:

$$
\begin{aligned}
& E_{i o, \beta}+E_{r o, \beta}=E_{t o, \beta}, \\
& E_{i o, \beta} \cos \zeta_{i}-E_{r o, \beta} \cos \zeta_{i}=\left[\left(N_{r}+i N_{i}\right) \cos \zeta_{t}+i N_{n} \sin ^{2} \zeta_{t}\right] E_{t o, \beta}, \\
& E_{i o, \alpha}+E_{r o, \alpha}=\left(N_{r}+i N_{i}\right) E_{t o, \alpha}, \\
& E_{i o, \alpha} \cos \zeta_{i}-E_{r o, \alpha} \cos \zeta_{i}=\cos \zeta_{t} E_{t o, \alpha} .
\end{aligned}
$$

The preceding equations may be solved to obtain the reflection coefficients for the two polarization configurations:

$$
\begin{aligned}
R_{T E, \perp}= & E_{r o, \beta} / E_{i o, \beta}=\frac{\cos \zeta_{i}-\left[\left(N_{r}+i N_{i}\right) \cos \zeta_{t}+i N_{n} \sin ^{2} \zeta_{t}\right]}{\cos \zeta_{i}+\left[\left(N_{r}+i N_{i}\right) \cos \zeta_{t}+i N_{n} \sin ^{2} \zeta_{t}\right]} \\
& =\frac{\cos \zeta_{i}-\left(N_{r} \cos \zeta_{t}+i N_{n}\right)}{\cos \zeta_{i}+\left(N_{r} \cos \zeta_{t}+i N_{n}\right)}, \\
R_{T E, l l} & =E_{r o, \alpha} / E_{r o, \beta}=\frac{\left(N_{r}+i N_{i}\right) \cos \zeta_{i}-\cos \zeta_{t}}{\left(N_{r}+i N_{i}\right) \cos \zeta_{i}+\cos \zeta_{t}} .
\end{aligned}
$$


Similarly, if we assume that the magnetic field of the refracted wave is transverse with respect to the ray direction (hereafter, this mode is referred to as TM mode), the reflection coefficients are then given by:

$$
\begin{aligned}
& R_{T M, \perp}=\frac{\left(N_{r}+i N_{i}\right) \cos \zeta_{i}-\varepsilon \cos \zeta_{t}}{\left(N_{r}+i N_{i}\right) \cos \zeta_{i}+\varepsilon \cos \zeta_{t}}, \text { and } \\
& R_{T M, / /}=\frac{\varepsilon \cos \zeta_{i}-\left(N_{r} \cos \zeta_{t}+i N_{n}\right)}{\varepsilon \cos \zeta_{i}-\left(N_{r} \cos \zeta_{t}+i N_{n}\right)} .
\end{aligned}
$$

Therefore, considering the two wave modes and two polarization configurations, four combinations are possible for the reflection coefficients: $\left(R_{T M, / /}, R_{T M, \perp}\right),\left(R_{T E, / /}, R_{T E, \perp}\right)$, $\left(R_{T E, / i}, R_{T M, \perp}\right)$, and $\left(R_{T M, / i}, R_{T E, \perp}\right)$. Each of these four combinations is a mathematically rational solution derived from the electromagnetic boundary condition when an absorptive medium is involved. However, only one among the four solutions should be computationally correct in the ray tracing calculation regarding the process of scattering of electromagnetic wave by a dielectric particle with absorption. It should be pointed out that the Maxwell equations subject to appropriate boundary conditions always have a unique solution, as is in the case of Mie theory. The explanation for the multiple solutions for the reflection coefficients is that electromagnetic boundary condition is locally imposed in the context of geometric optics, which allows the treatment of the inhomogeneous refracted wave as a refracted ray, thereby providing the basis for a raytracing procedure. Accordingly, we are looking for the pair of reflection coefficients that provide the best numerical agreement with the exact solution in scattering computation.

To identify the computationally correct pair of reflection coefficients among the preceding four combinations, we consider the scattering geometry in Fig. 2 for a strongly absorptive sphere with a size much larger than incident wavelength. In this case, the 
scattered field is composed primarily of the contributions from diffraction and external reflection. The incident energy associated with the area element $d s$ on the projected area is scattered in angular element $\sin \theta d \theta d \varphi$ in which $\theta$ is scattering angle. The scattered intensity at a distance $r$ that is far away from the particle is given by

$$
I_{s}=I_{o} \frac{|R|^{2}}{r^{2}}\left|\frac{d s}{\sin \theta d \theta d \varphi}\right|=I_{o} \frac{|R|^{2} a^{2}}{4 r^{2}},
$$

where $a$ is the radius of the sphere; $I_{o}$ and $I_{s}$ are incident and scattered intensity, respectively; $R$ is reflection coefficient without a specification of wave mode and polarization configuration. To derive Eq.(14), we have used two relationships given by $d s=a^{2} \cos \zeta_{i} \sin \zeta_{i} d \zeta_{i} d \varphi$ and $\theta=\pi-2 \zeta_{i}$. On the other hand, according to the definition of scattering phase function denoted by $P$, one may define the following relationship:

$$
I_{s}=\frac{\sigma_{s}}{4 \pi r^{2}} P I_{o},
$$

where $\sigma_{s}$ is the scattering cross section. Therefore, after the two polarization configurations are accounted for, the contribution of the external reflection to phase function may be obtained from comparison of Eqs.(14) and (15):

$$
\begin{aligned}
P^{r}(\theta) & =\frac{1}{2} \frac{\left(\left|R_{l i}\right|^{2}+\left|R_{\perp}\right|^{2}\right) \pi a^{2}}{\sigma_{s}} \\
& =\frac{1}{2} \frac{\left(\left|R_{i i}\right|^{2}+\left|R_{\perp}\right|^{2}\right)}{Q_{e}-Q_{a}},
\end{aligned}
$$

where $Q_{e}$ and $Q_{a}$ are the extinction efficiency and absorption efficiency, respectively. The value of $Q_{e}$ is 2 since the particle is very large whereas $Q_{a}$ is determined by the ratio of refracted energy to the incident energy associated with the projected area. Note that the 
divergence factor introduced by van de $\mathrm{Hulst}^{25}$ is absent in Eq.(16) because the rays that transmit through the sphere are ignored here.

An improved diffraction formulation has been given by Yang and $\mathrm{Liou}^{26}$ that, unlike the conventional method that is limited to scattering angle smaller than $90^{\circ}$, can be applied the entire scattering angular region. When the improved method is applied to the diffraction by a sphere, the scattering amplitude matrix ${ }^{25}$ is given by

$$
\left(\begin{array}{ll}
S_{2} & S_{3} \\
S_{4} & S_{1}
\end{array}\right)=\frac{(k a)^{2}}{4} \frac{2 J_{1}(k a \sin \theta)}{k a \sin \theta}\left(\begin{array}{cc}
\cos \theta(1+\cos \theta) & 0 \\
0 & 1+\cos \theta
\end{array}\right)
$$

where $J_{1}$ is the Bessel function of the first kind. The phase function that includes the contribution from diffraction and external reflection can be obtained from Eqs.(16) and (17) as follows:

$$
P(\theta)=\frac{(k a)^{2}}{8\left(Q_{e}-Q_{a}\right)}\left[\frac{2 J_{1}(k a \sin \theta)}{k a \sin \theta}\right]^{2}\left(1+\cos ^{2} \theta\right)(1+\cos \theta)^{2}+\frac{1}{2} \frac{\left(\left|R_{/ /}\right|^{2}+\left|R_{\perp}\right|^{2}\right)}{Q_{e}-Q_{a}} .
$$

In addition to the phase function, the polarization configuration should be used to identify the correct combination of reflection coefficients. When the incident radiation is unpolarized, the polarization feature of scattered radiation can be specified by the degree of linear polarization (DLP). When scattered radiation is composed of diffraction and external reflection, the DLP associated with the phase function in Eq.(18) may be given as follows:

$$
\begin{gathered}
\operatorname{DLP}(\theta)=\frac{I_{\perp}^{d}(\theta)-I_{l /}^{d}(\theta)+I_{\perp}^{r}(\theta)-I_{/ /}^{r}(\theta)}{I_{\perp}^{d}(\theta)+I_{/ i}^{d}(\theta)+I_{\perp}(\theta)+I_{f i}^{r}(\theta)} \\
=\frac{(k a)^{2}\left[2 J_{1}(k a \sin \theta) / k a \sin \theta\right]^{2}(1+\cos \theta)^{2}\left(1-\cos ^{2} \theta\right)+4\left(\left|R_{\perp}\right|^{2}-\left|R_{/ /}\right|^{2}\right)}{(k a)^{2}\left[2 J_{1}(k a \sin \theta) / k a \sin \theta\right]^{2}(1+\cos \theta)^{2}\left(1+\cos ^{2} \theta\right)+4\left(\left|R_{\perp}\right|^{2}+\left|R_{/ /}\right|^{2}\right)}
\end{gathered}
$$


$I_{\perp}^{d}$ and $I_{\perp}^{r}\left(I_{/ /}^{d}\right.$ and $\left.I_{l i}^{r}\right)$ are the scattered intensity associated with diffraction and external reflection, respectively, for the case that the electric field is polarized along a direction that is perpendicular (parallel) to the scattering plane.

Figure 3 shows the phase function and DLP computed from Mie theory and the geometric optics method at a wavelength of $12 \mu \mathrm{m}$. The Mie computational code developed by Wiscombe ${ }^{27}$ is used in this study. Since the size parameter is extremely large $(\chi=1047.2)$, the scattered energy at a large scattering angle (approximately $20^{\circ}$ in this case) is primarily from the reflection of incident rays. Through comparison of the geometric optics solution to the Mie results, we can identify the correct reflection coefficient pair. Evidently, the reflection coefficient pair $\left(R_{T M, / /}, R_{T E, \perp}\right)$ produces a phase function and DLP consistent with the Mie solution at large scattering angels where the external reflection dominates. Since the Mie solution for DLP is positive for the entire scattering domain, the reflection of incident wave with vertical polarization configuration is much larger than the reflection for the case when the polarization is parallel to scattering plane. For this reason, if we incorrectly apply the reflection coefficient $R_{T M, \perp}$ for the incident wave polarized perpendicularly with respect to scattering plane, substantial errors can be produced for both phase function and DLP, as is evident from Fig.3.

Figure 4 is similar to Fig.3, except that the wavelength is $11 \mu \mathrm{m}$ and the real part of the refractive index is close to unity (see Fig. 1). It can been seen that the phase function values at large scattering angles at $11 \mu \mathrm{m}$ are much smaller than the corresponding results at $12 \mu \mathrm{m}$ since external reflection is weaker for the former wavelength. However, the correctness of reflection coefficients used in the computation 
is also critical to the phase function and DLP even in the optically tenuous case. As a matter of fact, the errors associated when the wrong wave mode is assumed for the refracted wave are even more pronounced for the results shown in Fig.4 than for those shown in Fig.3. From these results, it is clear that the correct wave mode must be used for the refracted wave in the derivation of the reflection coefficients regardless of the magnitude of the real part of refractive index whenever the particle is strongly absorptive.

The comparison of the ray-tracing method to Mie theory for the phase function of spheres has been presented by Liou and Hansen ${ }^{28}$ for a polydispersive case having an assumed particle size distribution. For a monodispersive case (a system composed of particles having a single size and shape), the comparison study has been presented by Macke et $a .^{29}$ for spheroids and finite circular cylinders at a near infrared wavelength, which shows that the applicable size parameter for the geometric optics method is approximately 60 . In addition, these authors pointed out that the size parameter required for a convergence of the exact solution and the geometric optics solution is larger for spheres than for nonspherical particles.

The results of this study suggest that the geometric optics method can be applied to much smaller size parameters when strong absorption is involved. Figure 5 shows the phase function computed from Eq.(18) in comparison with the Mie solutions. Evidently, when the size parameter is larger than approximately 30 , the geometric optics solution can be used to approximate the exact solution. For size parameters larger than 50 , the geometric optics solution essentially converges to the exact solution in the side and backscattering directions. 
From the preceding discussions associated Figs. 3, 4 and 5, it is clear that TM and TE modes should be applied to the components of electric field that are parallel and perpendicular to the incident plane, respectively. By using this combination of wave modes, it is straightforward to derive the refracted wave on the basis of electromagnetic boundary conditions. Through application of the $\left(T M_{/ /}, T E_{\perp}\right)$ wave mode to the refracted wave, we can obtain the refracted electric field as follows:

$$
\begin{aligned}
& \vec{E}_{t, o}=E_{t, o, \alpha} \hat{\alpha}_{t}+E_{t, o, \beta} \hat{\beta}+E_{t, o, \gamma} \hat{e}_{t}, \\
& \left(\begin{array}{l}
E_{t o, \alpha} \\
E_{t o, \beta} \\
E_{t o, \gamma}
\end{array}\right)=\left(\begin{array}{cc}
T_{\alpha} & 0 \\
0 & T_{\beta} \\
T_{\gamma} & 0
\end{array}\right)\left(\begin{array}{l}
E_{i o, \alpha} \\
E_{i o, \beta}
\end{array}\right),
\end{aligned}
$$

where

$$
\begin{aligned}
& T_{\alpha}=\frac{2\left(N_{r}+i N_{i}\right) \cos \zeta_{i}}{\varepsilon \cos \zeta_{i}+\left(N_{r} \cos \zeta_{t}+i N_{n}\right)}, \\
& T_{\beta}=\frac{2 \cos \zeta_{i}}{\cos \zeta_{i}+\left(N_{r} \cos \zeta_{t}+i N_{n}\right)}, \\
& T_{\gamma}=\frac{i 2 N_{n} \sin \zeta_{t} \cos \zeta_{i}}{\varepsilon \cos \zeta_{i}+\left(N_{r} \cos \zeta_{t}+i N_{n}\right)} .
\end{aligned}
$$

Note that in Eqs.(20a)-(20e) the dependence of these parameters on the wave mode is not specified explicitly. It should be pointed out that the transmission matrix in the conventional ray-tracing scheme is a $2 \times 2$ matrix. When absorption is involved, the transmission matrix becomes a $3 \times 2$ matrix. When absorption of the medium is absent, $T_{\gamma}$ is reduced to zero because $N_{n}$ is zero and Eqs.(20a)-(20e) are reduced to the conventional Fresnel refraction coefficients. 


\section{Asymptotic Solution for Scattering and Absorption Properties of Large Polyhedral Particles with Strong Absorption}

\section{A. Physical Basis for Asymptotic Solution}

In the previous section, it is shown that the conventional geometric optics approach in terms of the superposition of diffraction and external reflection can well approximate the Mie solution for the spherical particle case when the size parameter is large and the particle is absorptive. Since the variation in the surface normal direction is continuous for a sphere, a continuous distribution of the externally reflected energy is obtained. However, this is not true if the particle geometry is a polyhedron such as a hexagonal plate or column, which is the basic geometric structure of ice crystals in cirrus clouds. In the numerical solution, there is a physically incorrect discontinuous distribution of scattered energy for any specific orientation of the particle.

For example, consider the scattering of radiation by a hexagonal ice column when the incident radiation is perpendicular to the $c$-axis of the particle and parallel to a line that connects two symmetric apexes of particle cross section. If the rays refracted into the particle are ignored due to absorption, the phase function can be written as follows:

$$
\begin{gathered}
P\left(\theta_{s}, \varphi_{s}\right)=\frac{3 k^{2} L a}{8 \pi \sigma_{s}}\left\{\frac{\sin \left[(k L / 2) \sin \theta_{s} \cos \varphi_{s}\right]}{(k L / 2) \sin \theta_{s} \cos \varphi_{s}} \frac{\sin \left[(\sqrt{3} k a / 2) \sin \theta_{s} \sin \varphi_{s}\right]}{(\sqrt{3} k a / 2) \sin \theta_{s} \sin \varphi_{s}}\right\}^{2} \\
\cdot\left(1+\cos ^{2} \theta_{s}\right)\left(1+\cos \theta_{s}\right)^{2}+\frac{\sqrt{3} \pi a L}{\sigma_{s}}\left[\delta\left(\varphi_{s}-\pi / 2\right)+\delta\left(\varphi_{s}+\pi / 2\right)\right] \\
\left.\delta\left(\theta_{s}-2 \pi / 3\right)\right]\left(\left|R_{T M, / /, \zeta_{i}=\pi / 6}\right|^{2}+\left|R_{T E, \perp, \zeta_{i}=\pi / 6}\right|^{2}\right), \\
\sigma_{s}=\sqrt{3} a L\left(2-\left|R_{T M, / / \zeta_{i}=\pi / 6}\right|^{2}-\left|R_{T E, \perp, \zeta_{i}=\pi / 6}\right|^{2}\right) .
\end{gathered}
$$

where $L$ and $a$ are the length and semi-width of hexagonal columns and $\delta(x)$ is the Dirac delta function. To avoid any possible confusion, in Eqs.(21a) and (21b) we use $\theta_{s}$ and $\varphi_{s}$ 
to indicate the scattering angle and the azimuthal angle of the scattering plane. Here the plane of $\varphi_{s}=0^{\circ}$ is the plane that contains incident direction and the $c$-axis of the particle. On the right side of Eq.(21a), the first term represents the contribution from diffraction and the second term represents the contribution of external reflection. Obviously, the second term is nonzero only at two angles as is expressed by the delta functions. This singularity is inherent in the conventional geometric optics method. The delta-reflection involved in Eq.(21) originates with the same physical mechanism of the forward delta-transmission that has been well explained by Takano and Liou ${ }^{12}$ and Mishchenko and Macke ${ }^{30}$. It should be pointed out that the singularity in Eq.(21b) could be overcome numerically in the ray-tracing calculation if the particles are randomly oriented in space. The average intensity is given by the scattered energy divided by the corresponding solid angle elements. The discontinuity may be avoided given proper resolution for the solid angle elements with sufficient orientations of the particles, i.e., with sufficiently random orientation.

To circumvent this particular disadvantage of the conventional method for a specific orientation, we apply the geometric optics method to solve for the internal field inside the particle following Yang and $\mathrm{Liou}^{31}$. The scattered far-field, extinction and absorption cross sections are given by

$$
\begin{aligned}
& \vec{E}^{s}(\vec{r})=\frac{k^{2} \exp (i k r)}{4 \pi r}(\varepsilon-1) \iint_{v}\left\{\vec{E}\left(\vec{r}^{\prime}\right)-\hat{r}\left[\hat{r} \cdot \vec{E}\left(\vec{r}^{\prime}\right)\right]\right\} \exp (-i k \hat{r} \cdot \vec{r}) d^{3} r^{\prime} \\
& \sigma_{e x t}=\operatorname{Im}\left[\frac{k}{\left|\vec{E}_{i}\right|}(\varepsilon-1) \iiint_{v} \vec{E}\left(\vec{r}^{\prime}\right) \vec{E}_{i}\left(\vec{r}^{\prime}\right)\right] d^{3} r^{\prime} \\
& \sigma_{a b s}=\frac{k}{\left|\vec{E}_{i}\right|^{2}} \varepsilon_{i} \iint_{v} \vec{E}\left(\vec{r}^{\prime}\right) \cdot \vec{E}_{i}\left(\vec{r}^{\prime}\right) d^{3} r^{\prime}
\end{aligned}
$$


The integrals involved in preceding equations may be calculated using the ray-by-ray integration (RBRI) algorithm ${ }^{31}$. From the RBRI calculation and Eqs. (22a)-(22c), the amplitude scattering matrix and extinction and absorption cross sections are given by:

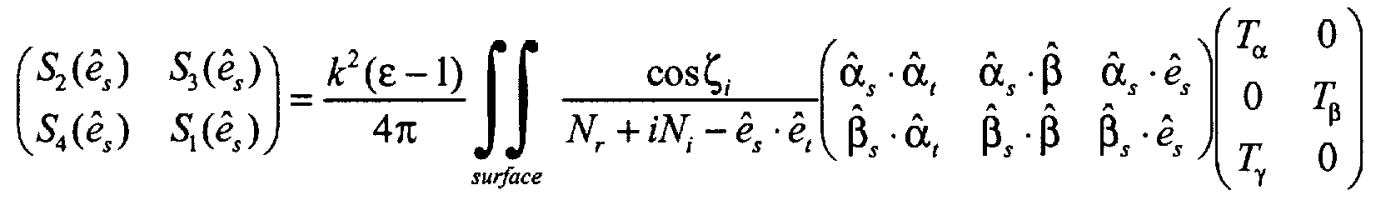

$$
\begin{aligned}
& \cdot\left(\begin{array}{cc}
\hat{\alpha}_{i} \cdot \hat{\alpha}_{o} & \hat{\alpha}_{i} \cdot \hat{\beta}_{s} \\
\hat{\beta} \cdot \hat{\alpha}_{o} & \hat{\beta} \cdot \hat{\beta}_{s}
\end{array}\right) \exp \left[-i k\left(\hat{e}_{i}-\hat{e}_{s}\right) \cdot \vec{r}^{\prime}\right]\left\{1-\exp \left[-k N_{i} l\left(\vec{r}^{\prime}\right)\right] \exp \left[i k\left(N_{r}-\hat{e}_{s} \cdot \hat{e}_{t}\right) l(\vec{r})\right]\right\} d^{2} r^{\prime},(23 \mathrm{a}) \\
& \sigma_{e x t}=\frac{2 \pi}{k^{2}} \operatorname{Re}\left[S_{2}\left(\hat{e}_{i}\right)+S_{2}\left(\hat{e}_{i}\right)\right], \\
& \sigma_{a b s}=\frac{1}{2} \iint_{\text {paricle surface }} \cos \zeta_{t} N_{r}\left(\left|T_{\alpha}\right|^{2}+\left|T_{\beta}\right|^{2}+\left|T_{\gamma}\right|^{2}\right)\left\{1-\exp \left[-2 k N_{i} l\left(r^{\prime}\right)\right]\right\} d^{2} r^{\prime},
\end{aligned}
$$

where $\hat{\beta}_{s}$ is a unit vector perpendicular to scattering plane; $\hat{e}_{s}$ is along scattering direction; $\hat{\alpha}_{s}=\hat{e}_{s} \times \hat{\beta}_{s} ; \quad \hat{\alpha}_{o}=\hat{e}_{i} \times \hat{\beta}_{s} ; \quad$ and $l\left(\vec{r}^{\prime}\right)$ is the ray penetration depth with the incident point at $\vec{r}^{\prime}$. The other variables involved are defined in the previous discussion. If the absorbing particle is large, it is expected that $\exp \left[-k N_{i} l\left(\vec{r}^{\prime}\right)\right] \rightarrow 0$. In this case, analytical expressions may be derived for the integration involved in Eqs. (23a) and (23c) if the surface of the particle is locally flat such as in the case of polyhedral geometry.

For a given flat face of the particle surface, Fresnel reflection does not vary with the location at the face because the incident angles are the same for all the points on this face. Thus, the following matrix is independent of the position of a point on a flat surface:

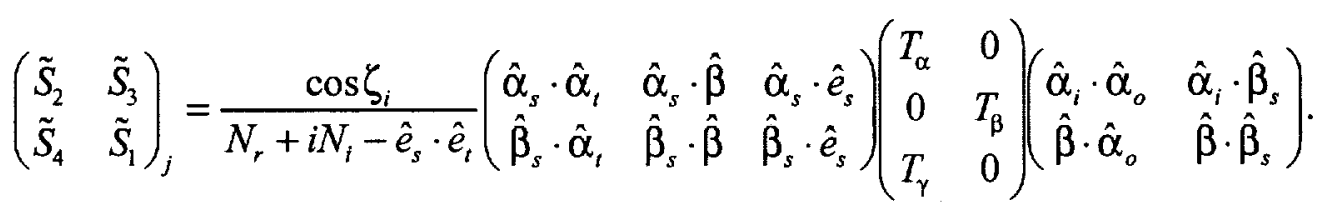


Therefore, the integral in Eq.(23a) should actually be evaluated only with respect to the phase variation over the local flat faces of the particle. For evaluation purposes, the surface of a polyhedral particle can be divided into a number of area elements shaped as parallelograms and triangles. For a parallelogram or a triangular face, the position vector of a point on this face is expressed by

$$
\vec{r}=\left\{\begin{array}{l}
\vec{r}_{o}+\xi \vec{r}_{1}+\eta \vec{r}_{2} \text { for parallelogram } \\
\vec{r}_{o}+\xi(1-\eta) \vec{r}_{1}+\eta \xi \vec{r}_{2} \text { for triangle }
\end{array},\right.
$$

where $\vec{r}_{o}$ is the position vector of the apex of the geometric shape, and $\bar{r}_{1}$ and $\bar{r}_{2}$ constitutes the neighboring two sides of the shape. The vector $\xi \in[0,1]$ and $\eta \in[0,1]$. From Eqs.(24) and (25), the integration of phase variation over a parallelogram area element yields

$$
\begin{aligned}
& D_{i}=\iint_{j a c e} \exp \left\{i k\left(\hat{e}_{i}-\hat{e}_{s}\right) \cdot \bar{r}_{j}\right\} d^{2} \vec{r}_{j} \\
& =\left|\vec{r}_{j, 1} \times \bar{r}_{j, 2}\right| \int_{0}^{1} \int_{0}^{1} \exp \left[i k\left(\hat{e}_{i}-\hat{e}_{s}\right) \cdot\left(\bar{r}_{j, 0}+\eta \bar{r}_{j, 1}+\xi \bar{r}_{j, 2}\right)\right] d \eta d \xi \\
& =\left|\bar{r}_{j, 1} \times \bar{r}_{j, 2}\right| \exp \left[i k\left(\hat{e}_{i}-\hat{e}_{s}\right) \cdot\left(\bar{r}_{j, 0}+\bar{r}_{j, 1} / 2+\bar{r}_{j, 2} / 2\right)\right] \\
& . \frac{\sin \left[k\left(\hat{e}_{i}-\hat{e}_{s}\right) \cdot \bar{r}_{j, 1} / 2\right]}{k\left(\hat{e}_{i}-\hat{e}_{s}\right) \cdot \vec{r}_{j, 1} / 2} \frac{\sin \left[k\left(\hat{e}_{i}-\hat{e}_{s}\right) \cdot \vec{r}_{j, 1} / 2\right]}{k\left(\hat{e}_{i}-\hat{e}_{s}\right) \cdot \bar{r}_{j, 1} / 2}
\end{aligned}
$$

Similarly, if the phase variation is integrated over a triangular area, it follows that

$$
\begin{gathered}
D_{i}=\iint_{\text {face } j} \exp \left\{i k\left(\hat{e}_{i}-\hat{e}_{s}\right) \cdot \vec{r}_{j}\right\} d^{2} \vec{r}_{j}, \\
=\left|\bar{r}_{j, 1} \times \bar{r}_{j, 2}\right| \frac{\exp \left[i k\left(\hat{e}_{i}-\hat{e}_{s}\right) \cdot \bar{r}_{j, 0}\right]}{i k\left(\hat{e}_{i}-\hat{e}_{s}\right) \cdot\left(\bar{r}_{j, 2}-\bar{r}_{j, 1}\right)}\left\{\exp \left[i k\left(\hat{e}_{i}-\hat{e}_{s}\right) \cdot \bar{r}_{j, 2} / 2\right] \frac{\sin \left[k\left(\hat{e}_{i}-\hat{e}_{s}\right) \cdot \bar{r}_{j, 2} / 2\right]}{k\left(\hat{e}_{i}-\hat{e}_{s}\right) \cdot \bar{r}_{j, 2} / 2}\right.
\end{gathered}
$$




$$
\left.-\exp \left[i k\left(\hat{e}_{i}-\hat{e}_{s}\right) \cdot \vec{r}_{j, 1} / 2\right] \frac{\sin \left[k\left(\hat{e}_{i}-\hat{e}_{s}\right) \cdot \vec{r}_{j, 1} / 2\right]}{k\left(\hat{e}_{i}-\hat{e}_{s}\right) \cdot \vec{r}_{j, 1} / 2}\right\}
$$

The scattering matrix that includes the contribution from all the faces can be written as

$$
\left(\begin{array}{ll}
S_{2}\left(\hat{e}_{s}\right) & S_{3}\left(\hat{e}_{s}\right) \\
S_{4}\left(\hat{e}_{s}\right) & S_{1}\left(\hat{e}_{s}\right)
\end{array}\right)=\frac{k^{2}(\varepsilon-1)}{4 \pi} \sum_{j} h\left(\hat{e}_{i} \cdot \hat{n}_{j}\right) D_{j}\left(\begin{array}{ll}
\tilde{S}_{2}\left(\hat{e}_{s}\right) & \tilde{S}_{3}\left(\hat{e}_{s}\right) \\
\tilde{S}_{4}\left(\hat{e}_{s}\right) & \tilde{S}_{1}\left(\hat{e}_{s}\right)
\end{array}\right)_{j}
$$

where $h\left(\hat{e}_{i} \cdot \hat{n}_{j}\right)$ is a step function that indicates whether the face is illuminated by incident radiation:

$$
h\left(\hat{e}_{i} \cdot \hat{n}_{j}\right)=\left\{\begin{array}{l}
1, \text { for } \hat{e}_{i} \cdot \hat{n}_{j}>0 \\
0, \text { for } \hat{e}_{i} \cdot \hat{n}_{j} \leq 0
\end{array} .\right.
$$

Once the amplitude scattering matrix is given, the calculation of phase function is straightforward on the basis of the definition presented by van de Hulst ${ }^{25}$. It should be pointed out that a special form of Eq.(27) have been given for hexagonal particle without an incorporation of the inhomogeneity effect of the refracted wave on the transmission matrix ${ }^{31}$. The result presented in this study is more general and can be applied to any polyhedron with strong absorption.

Since the multiple scattering calculation is based on the solution of the radiative transfer equation, the most important parameter of concern is the single-scattering albedo rather than extinction or absorption cross section. In particular, in remote sensing applications, a pre-described optical thickness is usually used in the calculation of lookup tables for the microphysical or optical properties of cloud particles. For this reason, in the present calculation we focus on the phase function and single-scattering albedo. The later parameter is defined as

$$
\tilde{\omega}=\frac{\sigma_{e x t}-\sigma_{a b s}}{\sigma_{e x t}}
$$


where the extinction and absorption cross sections, $\sigma_{e x t}$ and $\sigma_{a b s}$ can be calculated from Eqs. (23b) and (23c).

\section{B. Numerical Results for Hexagonal Ice Plates and Columns}

As an application for the asymptotic solution for light scattering by a strongly absorptive particle, we present numerical results for ice crystals composed of hexagonal plates and solid columns. A hexagonal shape is selected here because this shape has been studied extensively for inference of cloud optical thickness and particle size in remote sensing applications (e.g., Minnis et al. ${ }^{32}$, Baum et al. ${ }^{33}$, Han et al. ${ }^{34}$ ).

Fig. 6 shows the comparison of the phase function computed by the FDTD method and the asymptotic theory (Eq. 27) for hexagonal ice crystals of two sizes: $2 \mathrm{a} / \mathrm{L}=25 \mu \mathrm{m}$ $125 \mu \mathrm{m}$ and $40 \mu \mathrm{m} / 40 \mu \mathrm{m}$, where $L$ and $a$ are the length and semi-width of the hexagonal crystal. A random orientation condition is assumed for ice crystals in both the asymptotic and the FDTD calculations. It can be seen from Fig. 6 that the asymptotic solution more closely approximates the FDTD results for the larger particle size of $40 / 40 \mu \mathrm{m}$. While one might infer that the asymptotic theory can be applied to hexagonal ice crystals with sizes larger than approximately $40 \mu \mathrm{m}$ in the infrared window region, the convergence between FDTD and asymptotic theory will, in fact, depend on magnitude of absorption. For large particles at IR wavelengths, the FDTD calculation is computationally expensive. Thus, the asymptotic theory provides an efficient way to estimate the phase function of large particle with strong absorption. This advantage of the asymptotic theory in the calculation of phase function may be useful in practice. For example, the parameterization of cloud longwave radiative properties requires the phase function information though the use of either an asymmetry factor or a scaling factor ${ }^{35}$, which can 
be provided by the asymptotic theory efficiently and accurately when the particle size is larger than approximately $40 \mu \mathrm{m}$.

Figure 7 shows a comparison of the single-scattering albedo computed from the FDTD and the asymptotic theory. In this diagram, the results computed for the equivalent spheres are also shown. Following Mitchell et al. ${ }^{36}$, Fu et al. ${ }^{37}$, and Grenfell and Warren ${ }^{39}$, we define the radius of the equivalent sphere for a hexagonal particle as follows:

$$
r_{e}=\frac{3}{4} \frac{V}{A}=\frac{3}{2} \frac{\sqrt{3} / 2 a L}{\sqrt{3} / 2 a+L},
$$

where $V$ is the volume of nonspherical particles, and $A$ is the projected area. Since strong absorption is assumed in the computation of the single-scattering albedo in the asymptotic theory, the result does not change with the variation of particle size. As a result, the asymptotic theory can lead to substantial errors in single-scattering albedo calculation for small and moderate particle sizes. However, from the trend of the FDTD solution, it is expected that a convergence between the FDTD and asymptotic results can be reached when the particle size is on the order of 200. Approaches developed by Fu et al.$^{37}$, Michell ${ }^{39}$, and Baran et $\mathrm{al}^{40}$ can be used to overcome the shortcomings of the asymptotic theory in calculating single-scattering albedo. It should be pointed out that these approaches cannot provide the information for the phase function. For this reason, a combination of these approaches (for the computation of single-scattering albedo) with the asymptotic theory (for the computation of phase function) may be more useful in practice.

Figures 8 and 9 show the phase functions of randomly oriented ice columns and plates in comparison with the phase function of equivalent spheres. At large scattering angles, the phase function values are essentially the same for the spherical and 
nonspherical particles. The external reflection becomes insensitive to particle geometry once the random orientation condition is assumed. However, for moderate particle sizes $\left(r_{e}=26.7 \mu \mathrm{m}\right)$, substantial differences may be noted for the phase function at scattering angles in the $10^{\circ}-60^{\circ}$ region for the two particle shapes.

Figure 10 shows the variation of the single-scattering albedo of hexagonal ice plates and columns that are randomly oriented in the infrared region between $8 \mu \mathrm{m}$ and $16 \mu \mathrm{m}$. Also shown are the real and imaginary parts of refractive index data compiled by Warren ${ }^{41}$. Evidently, the variational pattern of the single-scattering albedo follows that of the real part of the refractive index. As we have mentioned, the real part of refractive index has a value close to unity in the vicinity of $11 \mu \mathrm{m}$, and the corresponding imaginary part is substantially large. Near $11 \mu \mathrm{m}$, the extinction of incident radiation is dominated by absorption, as is known as the Christiansen effect ${ }^{23}$. As a result, a minimum is noted in the single-scattering albedo. From Fig.10, it can also be seen that the single-scattering albedo for hexagonal particles is larger than for spheres. This feature has been noted in the comparison of the FDTD solution with the equivalent spherical results that are shown in Fig. 7.

It is a common practice to assume ice crystals are randomly oriented in space. However, in the atmosphere specific orientations are preferred for large plates and columns. For plates in an environment of a low Reynolds number, such as in a typical midlatitude cirrus cloud, the $a$-axis (the hexagonal asymmetric axis) of the particle tends to face vertically whereas the $c$-axis tends to be oriented horizontally. In other words, the crystals tend to align themselves so as to reach a stable state in its falling process. At visible wavelengths, the effect of preferred particle orientation on the phase function has 
been discussed by Takano and $\mathrm{Liou}^{42}$ and Rockwitz ${ }^{43}$. As pointed out in Section 3.A, the application of a conventional ray tracing technique will produce a discontinuity of the scattered energy and consequently cannot produce a continuous phase function. This shortcoming is avoided with the present analytical asymptotic theory. When preferred orientations are assumed for ice crystals, the phase function depends not only on the scattering angle but also on the scattering azimuthal angle, i.e., on which scattering plane the scattered field is observed. Figure 11 shows the geometry for the scattering configuration related to a plate oriented with its $c$-axis aligned vertically. The principal plane $\left(\varphi_{s}=0^{\circ}\right)$ is defined as the plane that contains the incident direction and zenith directions. The plane that is normal to the incident direction is denoted by the dotted ellipse whereas an arbitrary scattering plane is denoted by the dashed ellipse in the diagram. On a given scattering plane, the positive scattering angle is measured, with a view along the incident direction, clockwise from the principal plane. For example, on the principal plane the scattering angle is positive if it is measured from forward scattering direction to the vertical zenith whereas the scattering angle is negative if it is measured from forward scattering direction to nadir view.

Figure 12 shows the phase function for hexagonal plates having a preferred orientation for four scattering azimuthal angles. Ice crystals are rotated randomly about their $c$-axis. The strong forward peak caused by diffraction can been seen for the four cases with different scattering azimuthal angles. For the case of $\varphi_{s}=0^{\circ}$, very strong scattering peaks may be noted at scattering angle of $120^{\circ}$ and $60^{\circ}$ for incident zenith angles of $30^{\circ}$ and $60^{\circ}$, respectively. The scattering maxima at $120^{\circ}$ and $60^{\circ}$ correspond to the specular reflection from the top faces of the plates. For $\varphi_{s}=0^{\circ}, 30^{\circ}$ and $60^{\circ}$, 
significant asymmetry in the phase function is observed in the scattering angle regions of $\left(0^{\circ}, 180^{\circ}\right)$ and $\left(-0^{\circ},-180^{\circ}\right)$. However, symmetry is observed for $\varphi_{s}=90^{\circ}$, as is expected. For plates with a preferred orientation, there are a number of ripple structures in the phase function that are caused by phase interference.

Figure 13 shows the phase function for ice columns with preferred orientations. The columns, randomly rotating about the zenith direction, are horizontally oriented but rotate randomly about their $c$-axis. The ripple structure noted in Fig. 12 for plates due to the phase interference is largely smoothed out in the case of oriented columns because the orientation of the particles in the column case is more random. Similarly to the case in plates, the specular reflection feature is apparent in the case where $\varphi_{s}=0^{\circ}$.

Figure 14 shows the single-scattering albedo corresponding to the phase functions in Figs. 12 and 13. Compared with the results for randomly oriented particles, the single scattering for both oriented plates and columns are smaller if the incident zenith angle is less than approximately than $60^{\circ}$. For plates, there is a pronounced peak in the singlescattering albedo at the incident zenith of $70^{\circ}$. This is thought to be due to the external reflection for the top face of the plate increasing with an increase of incident zenith angle. However, when this angle approaches $90^{\circ}$, the projected area of the top face is essentially zero and the external reflection from the side faces, along with diffraction, dominates the scattered field.

\section{Summary and Conclusions}

An asymptotic solution in an analytical format is presented for the scattering properties of a general polyhedral particle with strong absorption. For scattering calculations involving nonspherical particles such as aerosols and ice crystals, we show 
that the transverse wave condition is not applicable to the refracted electromagnetic wave when absorption is involved. In the geometric optics solution for wave propagation in an absorbing dielectric medium, either the TM wave condition (i.e., where the magnetic field of the refracted wave is transverse with respect to the wave direction) or the TE wave condition (i.e., where the electric field is transverse with respect to the propagating direction of the wave) may be assumed for the refracted wave to satisfy the electromagnetic boundary condition. The wave mode assumed for the refracted wave affects both the reflection and refraction coefficients. As a result, a nonunique solution for these coefficients is derived from the electromagnetic boundary condition. Through comparison of the asymptotic geometric optics solution to results determined from Mie theory for absorbing spheres, it is shown that TM and TE wave modes should be applied to two polarized components that are parallel and perpendicular to the incident plane, respectively. In this study we have identified the appropriate solution for the Fresnel reflection coefficients in the ray tracing calculation. We present the $3 \times 2$ refraction or transmission matrix that completely accounts for the inhomogeneity of the refracted wave in an absorbing medium. Based on the Fresnel coefficients for an absorbing medium, we derive the asymptotic solution in an analytical format for the scattering properties of a general polyhedral particle. Numerical results are presented for hexagonal plates and columns with both preferred and random orientation. The asymptotic theory produces reasonable accuracy in the phase function calculations in the infrared window region (wavelengths near $10 \mu \mathrm{m}$ ) if the particle size is on the order of $40 \mu \mathrm{m}$ in diameter or larger. 
However, since strong absorption is assumed in the computation of the singlescattering albedo in the asymptotic theory, the single scattering albedo does not change with variation of the particle size. As a result, the asymptotic theory can lead to substantial errors in the computation of single-scattering albedo for small and moderate particle sizes. However, from comparison of results with the FDTD solution, it is expected that a convergence between the FDTD results and the asymptotic theory results can be reached when the particle size approaches $200 \mu \mathrm{m}$.

For two infrared wavelengths at 11 and $12 \mu \mathrm{m}$, we show that the phase function at side-scattering and backscattering angles is insensitive to particle shape if the random orientation is assumed. For preferred orientations, however, we show that the phase functions for plates and columns are significantly different. Additionally, when a preferred two-dimensional orientation condition is assumed, the phase function is observed to have a strong dependence on scattering azimuthal angle. Moreover, numerical results show that the single-scattering albedo has a very strong dependence on the inclination angle of incident radiation with respect to the rotating axis for the preferred particle orientations.

This research has been supported by a grant of NASA's MODIS project and partially by the Office of Naval Research. 


\section{References}

1. M. I. Mishchenko, J. W. Hovenier, and L. D. Travis, Light Scattering by Nonspherical Particles: Theory, Measurements, and Applications (Academic Press, San Diego, Cali., 1999).

2. E. M. Purcell and C. R. Pennypacker, 'Scattering and absorption of light by nonspherical dielectric grains," Astrophys. J. 186, 705-714, 1973.

3. B. T. Draine and P. J. Flatau, "Discrete-dipole approximation for light calculations," J. Opt. Soc. Am. A 11, 1491-1499.

4. P. Yang and K. N. Liou, "Finite-difference time domain method for light scattering by small ice crystals in three-dimensional space," J. Opt. Soc. Am. A 13, 2072-2085 (1996).

5. W.-B. Sun, Q. Fu, and Z. Chen, "Finite-difference time-domain solution of light scarttering by dielectric particles with a perfectly matched layer absorbing boundary condition," Appl. Opt. 38, 3141-3151 (1999).

6. G. Mie, "Beitrage zur Optik truber Medien, speziell kolloidaler Metallosungen," Ann. Phys. 25, 377-445 (1908).

7. S. Asano and M. Sato, "Light scattering by randomly oriented spheroidal particles," Appl. Opt. 19, 962-974 (1983)

8. V. G. Farafonov, N. V. Voshchinnikov, and V. V. Somsikov, "Light scattering y core-mantle spheroidal particles," Appl. Opt. 35, 5412-5426 (1996),

9. M. I. Mishchenko, "Light scattering by randomly oriented axially symmetric particles," J. Opt. Soc. Am. A 8, 871-882 (1991). 
10. M. I. Mishchenko, "Light scattering by size-shape distributions of randomly oriented axially symmetric particles of a size comparable to a wavelength," Appl. Opt. 32, 623-625 (1993).

11. M. I. Mishchenko, and A. Macke, "How big should hexagonal ice crystals be to produce halos?" Appl. Opt. 38, 1626-1629 (1999).

12.Y. Takano and K. N. Liou, "Solar radiative transfer in cirrus clouds. I. Singlescattering and optical properties of hexagonal ice crystals," J. Atmos. Sci. 46, 3-19 (1989).

13. A. Macke, "Scattering of light by polyhedral ice crystals," Appl. Opt. 32, 2780-2788 (1993).

14. J. A. Lock, "Ray scattering by an arbitrarily oriented spheroid. I. Diffraction and specular reflection, II. Transmission and cross-polarization effects," Appl. Opt. 35, 500-531 (1996).

15. K. Muinonen, K. Lumme, J.. Peltoniemi, and W. M. Irvine, "Light scattering by randomly oriented crystals," Appl. Opt. 28, 3051-3060 (1989).

16. A. Arking and J. D. Childs, "Retrieval of cloud cover parameters from multispectral satellite images," J. Climate and Appl. Meteoro. 24, 322-333 (1985).

17. M. D. King, Y. J. Kaufman, W. P. Menzel, and D. Tanre, "Remote sensing of cloud, aerosol, and water vapor properties from the Moderate Resolution Imaging Spectrometer (MODIS),", IEEE Trans. Geosci. Remote Sensing, 30, 2-26 (1992).

18. W. L. Smith, H. E. Revercomb, R. O. Knuteson, F. A. Best, R. Dedecker, H. B. Howell, and H. M. Woolf, Cirrus cloud properties derived from high spectral 
resolution infrared spectrometry during FIRE II. Part I: The high resolution interferometer sounder (HIS) systems," J. Atmos. Sci., 52, 4238-4245 (1995).

19. J. A. Stratton, Electromagnetic Theory, (McGraw-Hill, New York, 1941).

20. M. Born and E. Wolf, Principles of Optics, (Pergamon, Oxford, 1970).

21. J. Zhang and $\mathrm{L}$. $\mathrm{Xu}$, "Light scattering by absorbing hexagonal ice crystals in cirrus clouds," Appl. Opt. 34, 5867-5874.

22. P. Yang and K. N. Liou, "Light scattering by hexagonal ice crystals: Comparison of finite-difference time domain and geometric optics models," J. Opt. Soc. Am. A 12, 162-176 (1995).

23. W. P. Arnott, Y. Y. Dong, and J. Hallett, Extinction efficiency in the infrared (2-18 $\mu \mathrm{m})$ of laboratory ice clouds: Observations of scattering minima in the Christiansen bands of ice," Appl. Opt. 34, 541-551 (1995).

24.C. F. Bohren and D. R. Huffman, Absorption and Scattering of Light by Small Particles, (Wiley, New York, 1983).

25. H. C. van de Hulst, Light Scattering by Small Particles, (Wiley, New York, 1957).

26.P. Yang and K. N. Liou, "Single-scattering properties of complex ice crystals in terrestrial atmosphere," Contr. Atmos. Phys. 71, 223-248 (1998).

27. W. J. Wiscombe, "Improved Mie scattering algorithms," Appl. Opt. 19, 1505-1509 (1980).

28.K. N. Liou and J. E. Hansen, "Intensity and polarization for single scattering by polydisperse spheres: A comparison of ray optics and Mie theory," J. Atmos. Sci., 28, 995-1004 (1971). 
29. A. Macke, M. I. Mishchenko, K. Muinonen, B. E. Carlson, "Scattering of light by large nonspherical particles: ray-tracing approximation versus T-matrix method," Opt. Lett. 20, 1934-1936 (1995).

30.M. I. Mishchenko and A. Macke, "Incorporation of physical optics effect and computation of the Legendre expansion for ray-tracing phase functions involving $\delta$ function transmission," J. Geophys. Res. 103, 1799-1805 (1998).

31.P. Yang and K. N. Liou, "Light scattering by hexagonal ice crystals: solution by a ray-by-ray integration algorithm," J. Opt. Soc. Am. A 14, 2278-2289 (1997).

32. P. Minnis, K. N. Liou, and Y. Takano, "Inference of cirrus cloud properties using satellite-observed visible and infrared radiances, I. Parameteriztion of radiance fields," J. Atmos. Sci. 50, 1279-1304 (1993).

33. B. A. Baum, R. F. Arduini, B. A. Wielicki, P. Minnis, and S.-C. Tsay, "Multilevel cloud retrieval using multispectral HIRS and AVHRR data: Nighttime oceanic analysis," J. Geophys. Res. 99, 5499-5514 (1994).

34. Q. Han, W. B. Rossow, J. Chou, K-S. Kuo, and R. M. Welch, "The effect of aspect ratio and surface roughness on satellite retrieval of ice-cloud properties," J. Quant. Spectrosc. Radiat. Transfer 63, 559-583 (1999).

35. M.-D. Chou, K-T. Lee, S.-C. Tsay, and Q. Fu, "Parameterization for cloud longwave scattering for use in atmosphere models," J. Climate, 12, 159-169 (1999).

36. D. L. Mitchell, A. Macke, Y. Liu, "Modeling cirrus clouds, II, Treatment of radiative properties," J. Atmos. Sci., 53, 2967-2988 (1996). 
37.Q. Fu, P. Yang, and W. B. Sun, "An accurate parameterization of the infrared radiative properties of cirrus clouds for climate models," J. Climate, 11, 2223-2237 (1998).

38. T. C. Grenfell and S. G. Warren, "Representation of a nonspherical ice particle by a collection of independent spheres for scattering and absorption of radiation," J. Geophys. Res. 104, 31697-31709 (1999).

39. D. L. Mitchell, "Parameterization of the Mie extinction and absorption coefficients for water clouds," J. Atmos. Sci., 57, 1311-1326 (2000).

40.A. J. Baran and S. Havemann, "Rapid computation of the optical properties of hexgonal columns using complex angular momentum theory," J. Quant. Spectroscopy Radiat. Transfer 63, 499-519 (1999).

41. S. Warren, "Optical constants of ice from the ultraviolet to the microwave," Appl. Opt. 23, 1206-1225 (1984).

42.Y. Takano and K. N. Liou, "Radiative transfer in cirrus clouds. II. Theory and computation of multiple scattering in an anisotropic medium," J. Atmos. Sci. 46, 20$36(1989)$.

43. K.-D. Rockwitz, "Scattering properties of horizontally oriented ice crystal columns in cirrus clouds," Appl. Opt. 28, 4103-4110 (1989). 


\section{Figure Captions}

Fig.1. Comparison of the inherent and apparent refractive indices for ice at 11 and $12 \mu \mathrm{m}$.

Fig.2 Scattering geometry for a large sphere with strong absorption.

Fig.3 Phase function and the degree of linear polarization computed from geometric optics in comparison with Mie solution at $\lambda=12 \mu \mathrm{m}$.

Fig.4 As the same as Fig. 3 except that the wavelength is $11 \mu \mathrm{m}$ in Fig.4

Fig.5. Comparison of the phase functions computed from Mie theory and the geometric optics method for three moderate sizes at wavelengths 11 and $12 \mu \mathrm{m}$.

Fig.6 Comparison of phase function computed from the FDTD technique and the present asymptotic theory for hexagonal ice crystals.

Fig.7 Comparison of the single-scattering albedo computed from the FDTD technique and the present asymptotic theory for hexagonal ice crystals. Also shown are the results computed for equivalent spheres.

Fig. 8 Comparison of phase function of hexagonal columns with that of equivalent spheres.

Fig.9 As for Fig.8, except for hexagonal plates.

Fig.10 The comparison of the single-scattering albedo values computed for hexagonal ice crystals and equivalent spheres.

Fig.11 Incident and scattering geometry for an ice crystal with a preferred orientation.

Fig.12 Phase function computed for hexagonal plates with preferred orientations.

Fig.13 As for Fig.12 except for columns.

Fig.14. The single-scattering albedo for ice crystals that have preferred orientations in comparison with the results for the randomly oriented case. 
Real Part of Refractive Index
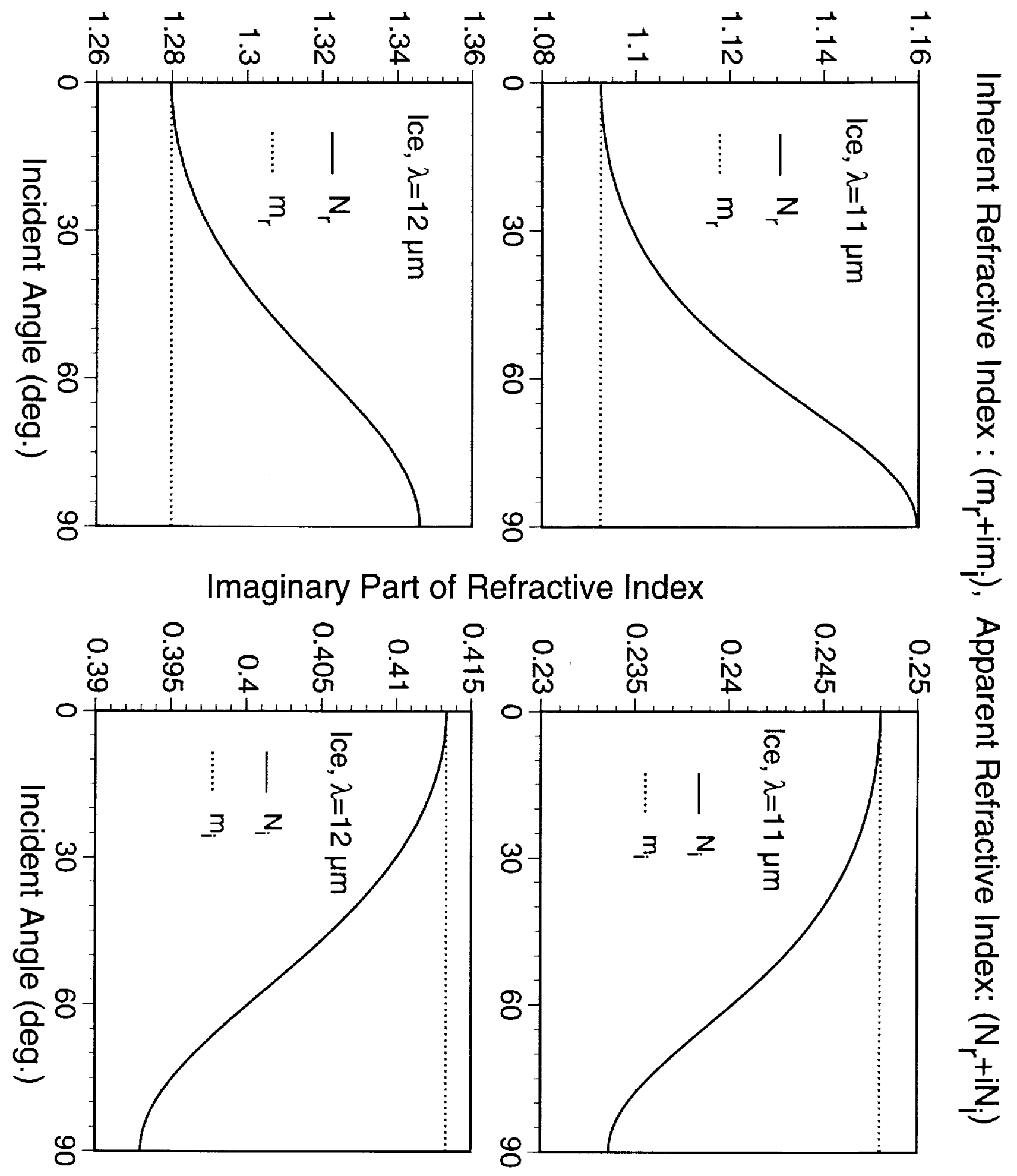


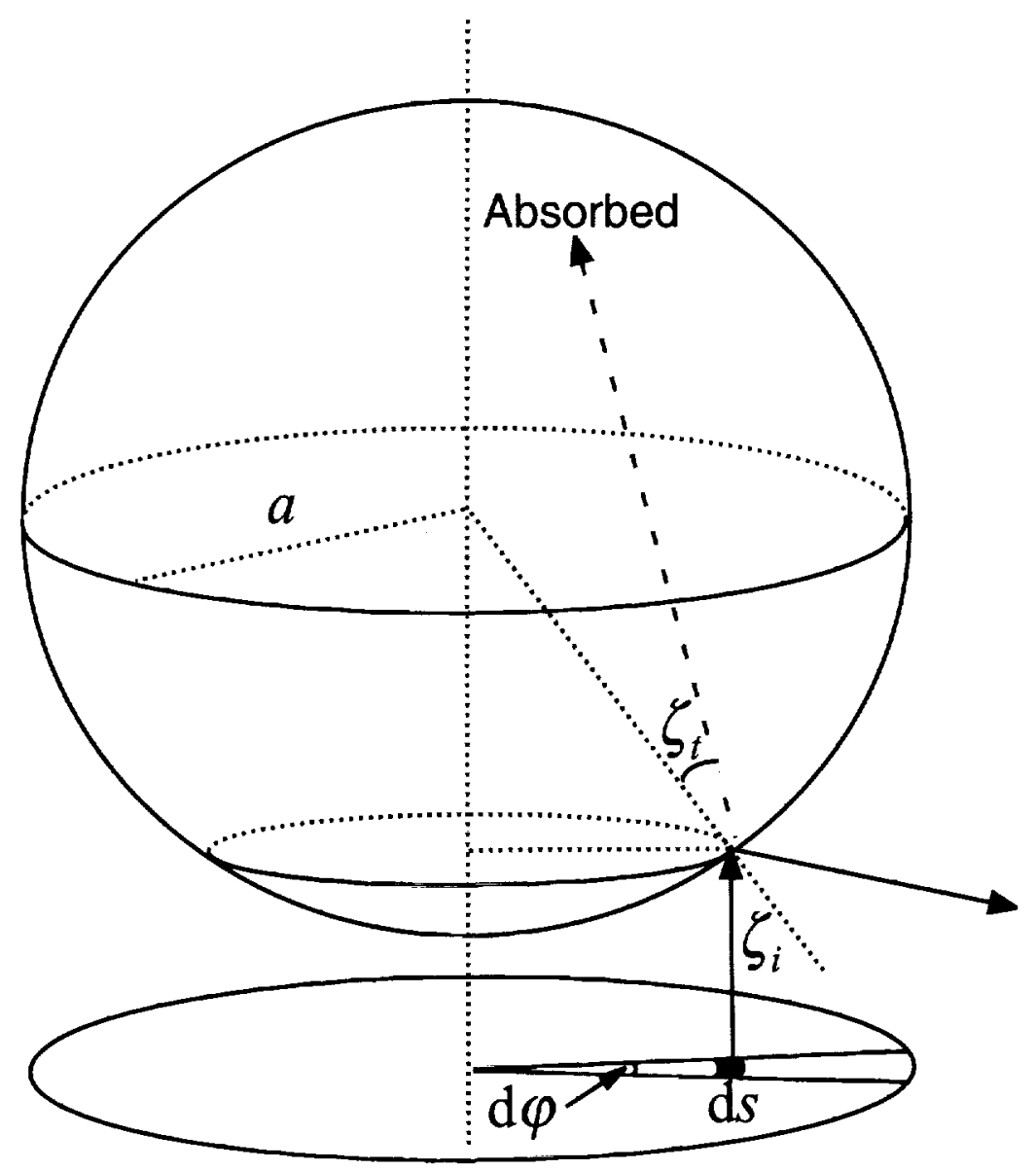

Fig.2 

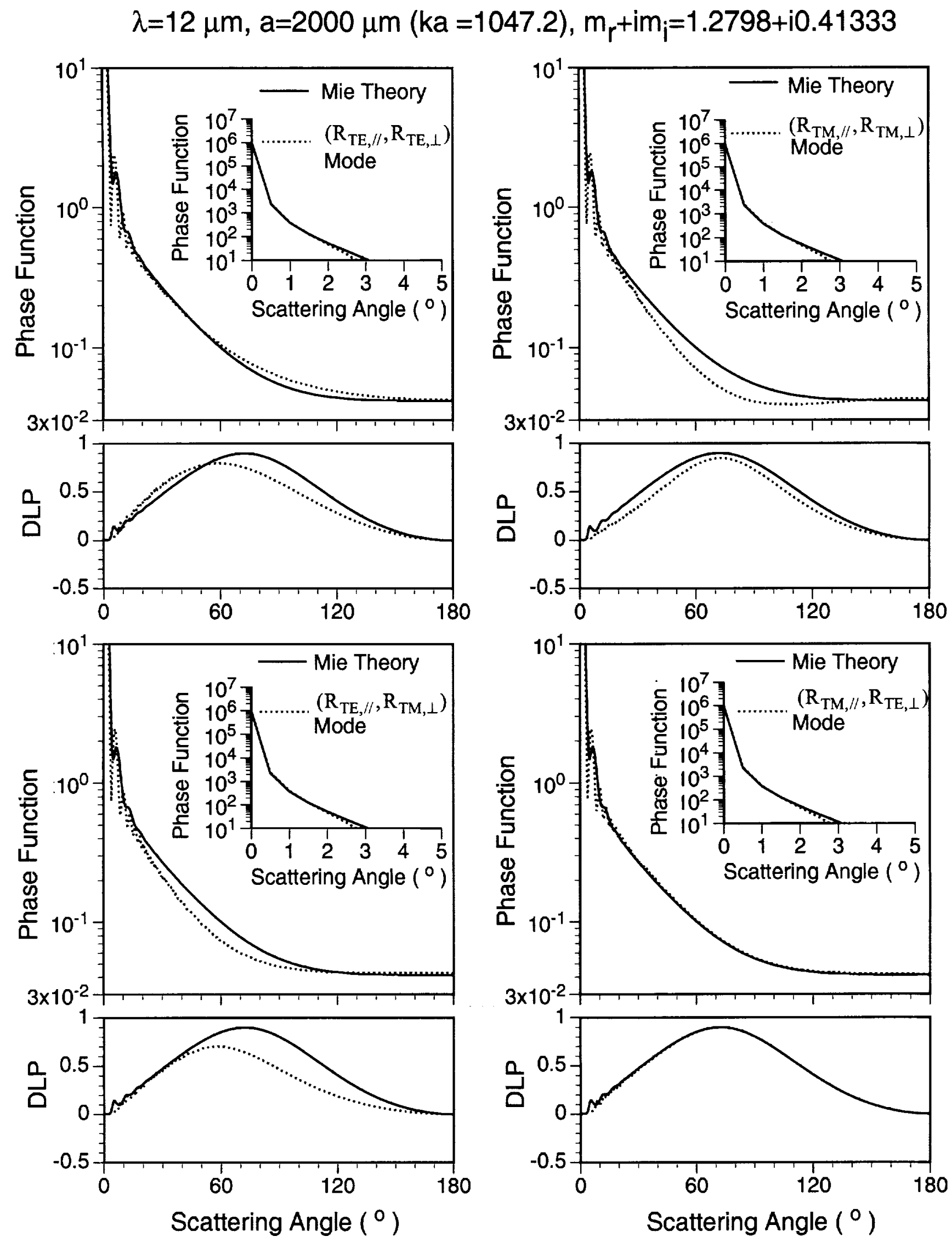

Fig.3 
$\lambda=11 \mu \mathrm{m}, a=2000 \mu \mathrm{m}(k a=1142.4), m_{r}+i m_{i}=1.0925+i 0.248$
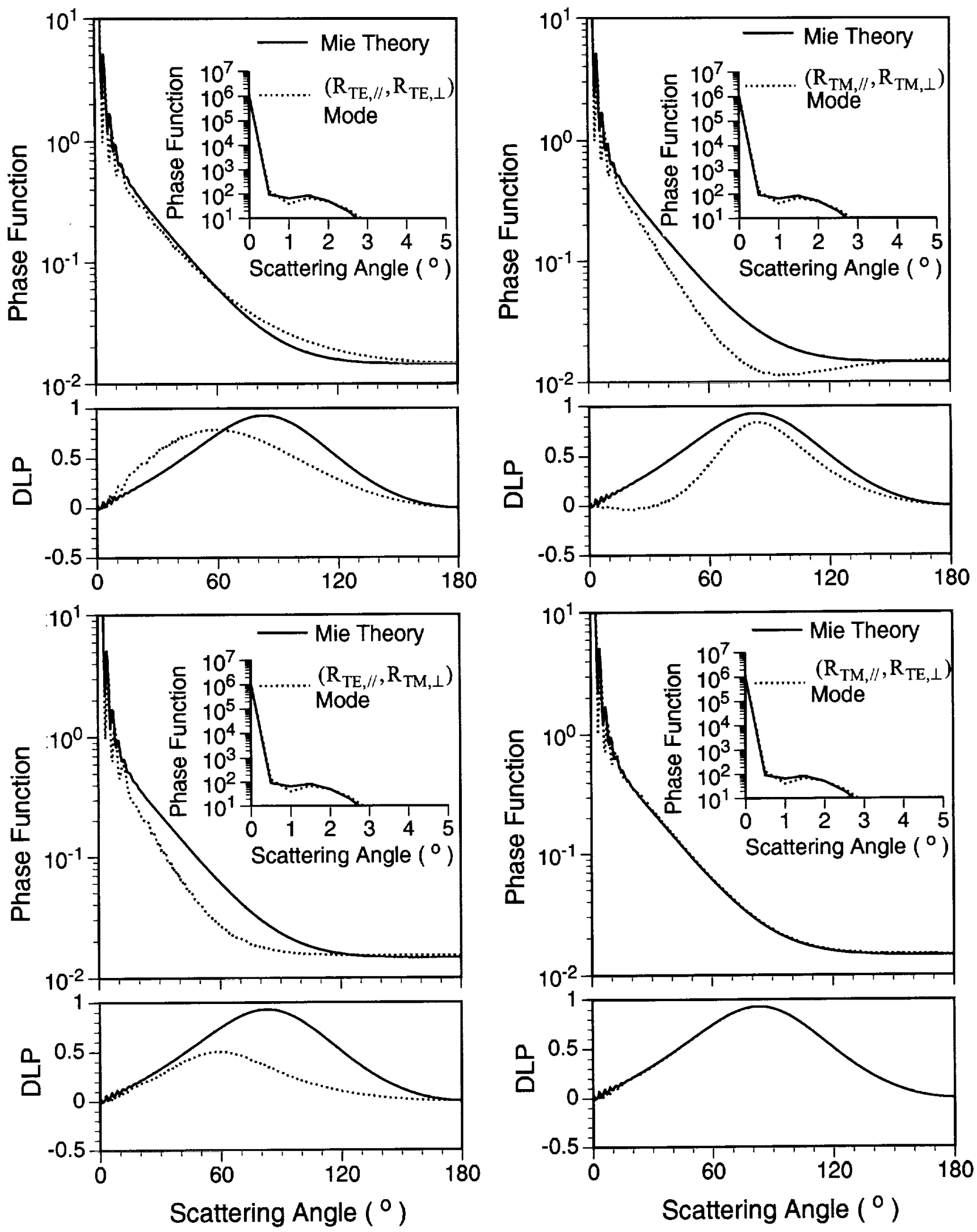

Fig.4 


\section{Phase Function}
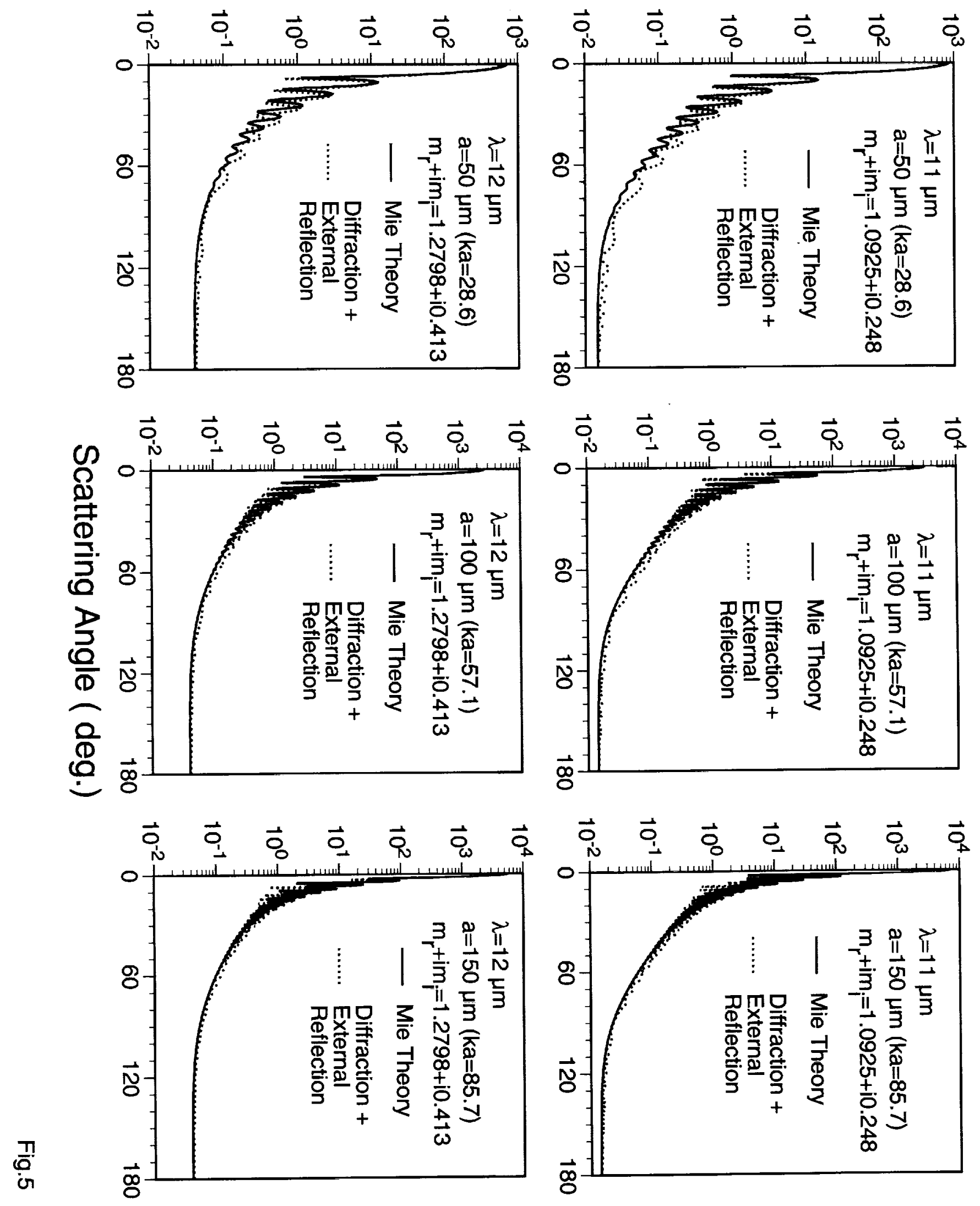

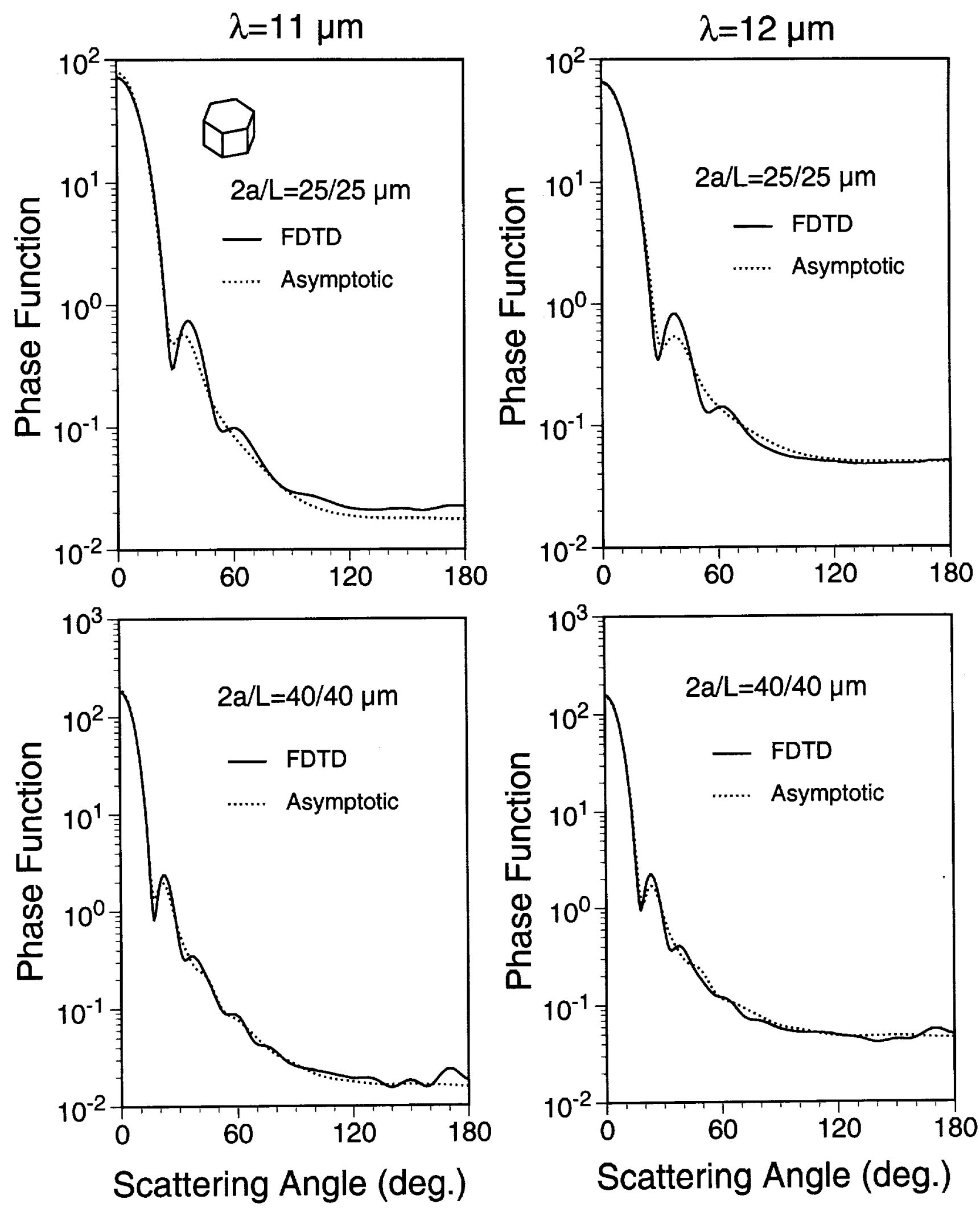

Fig.6 


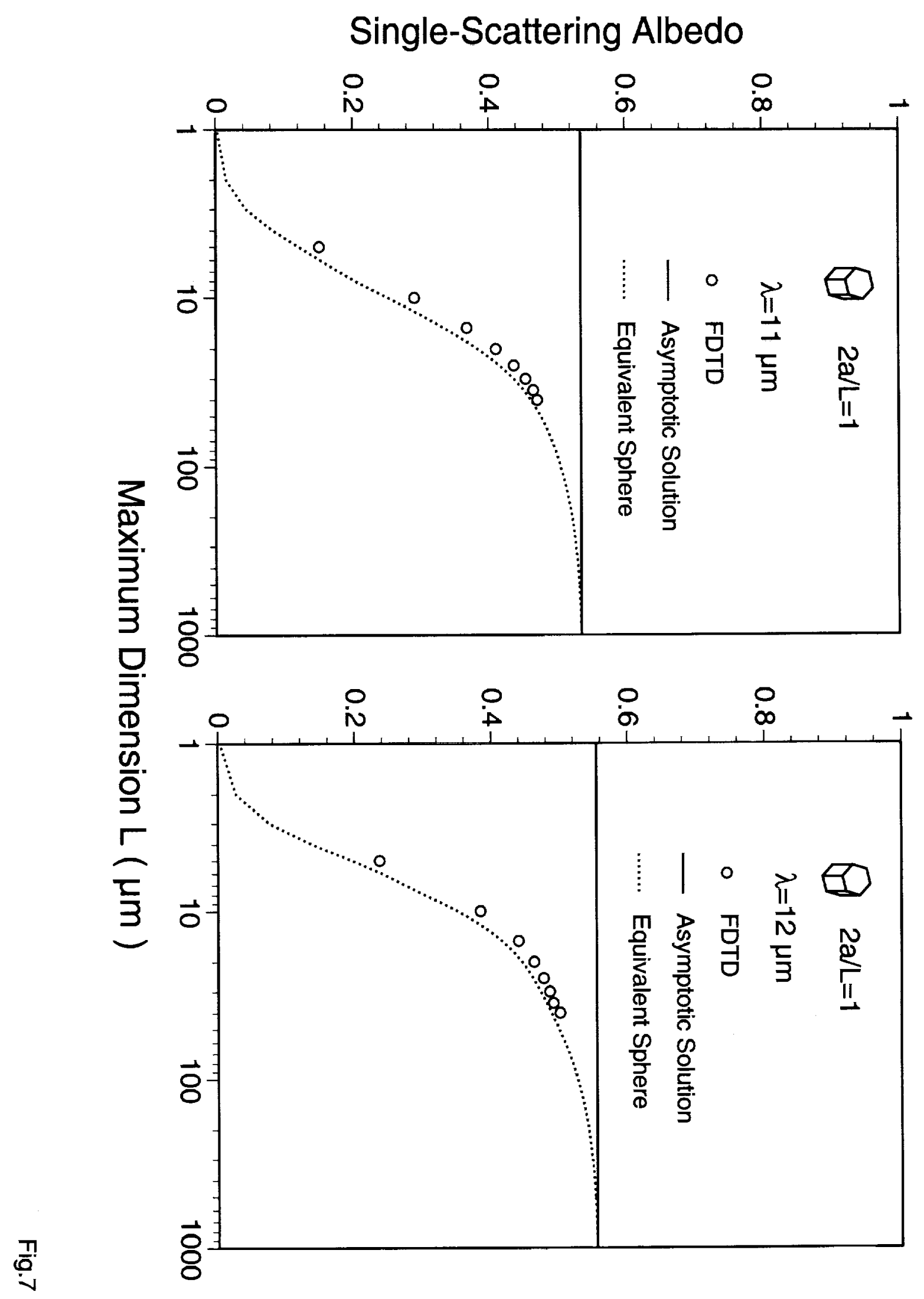



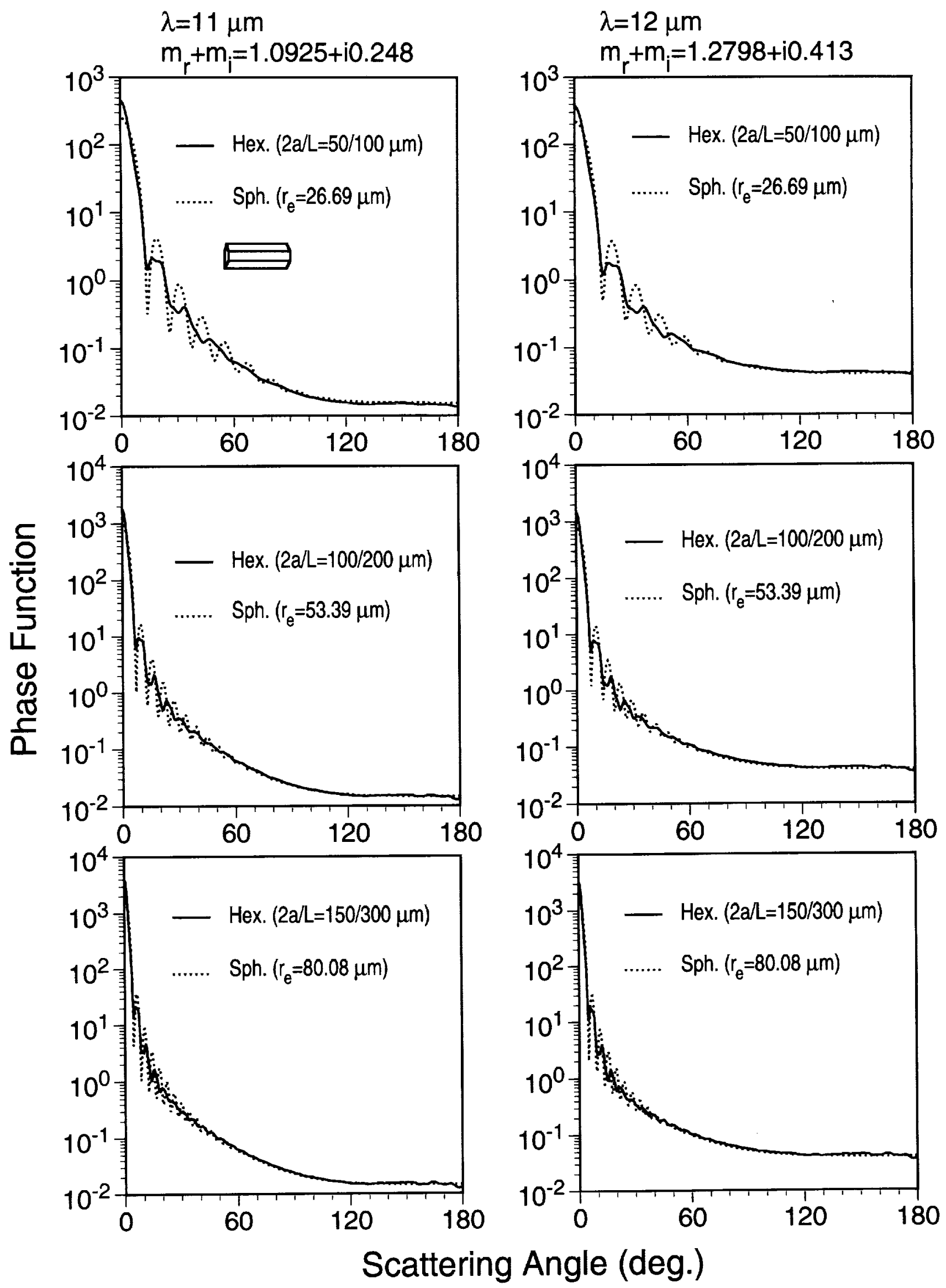

Fig.8 

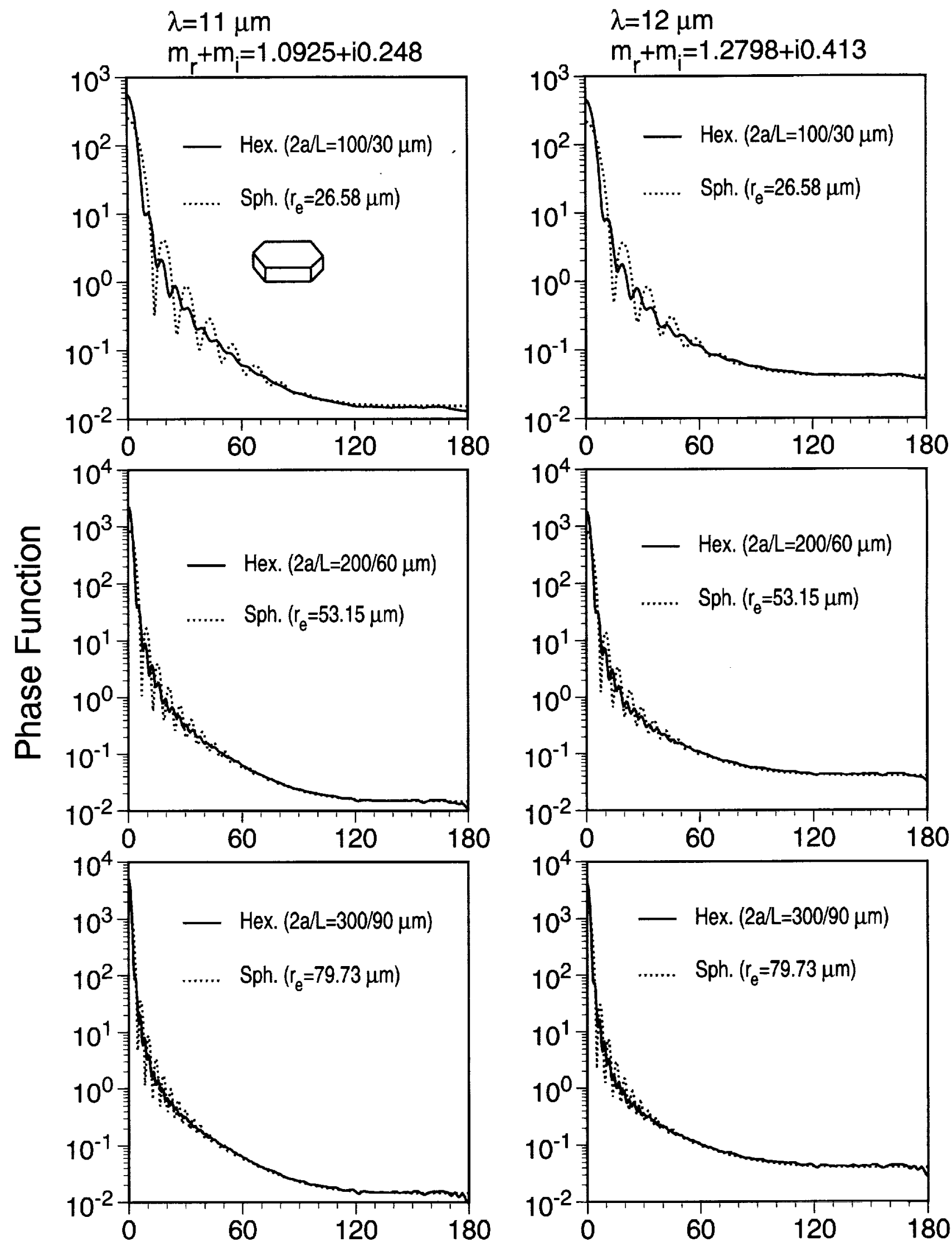

Scattering Angle (deg.)

Fig.9 


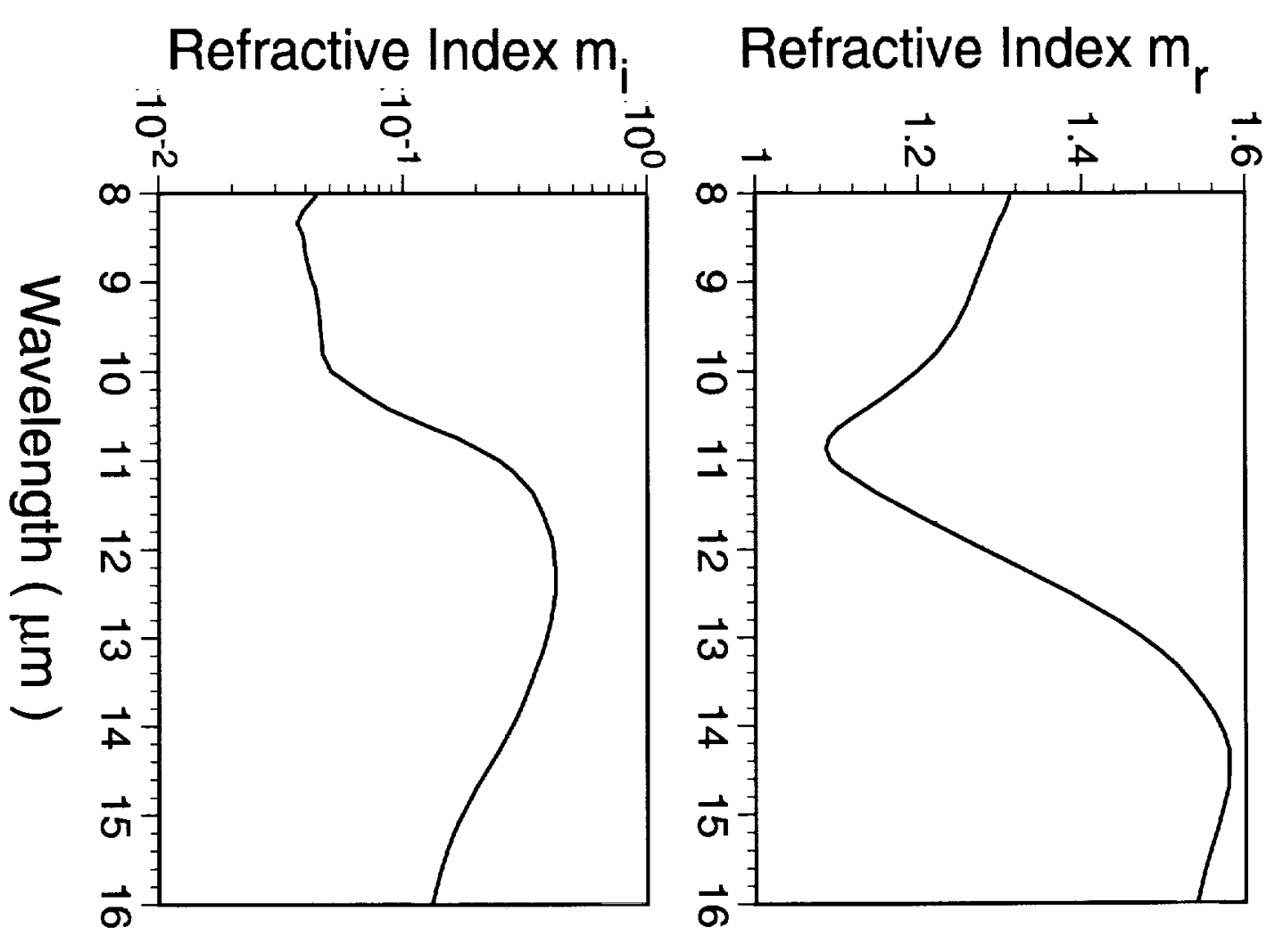

Single-Scattering Albedo
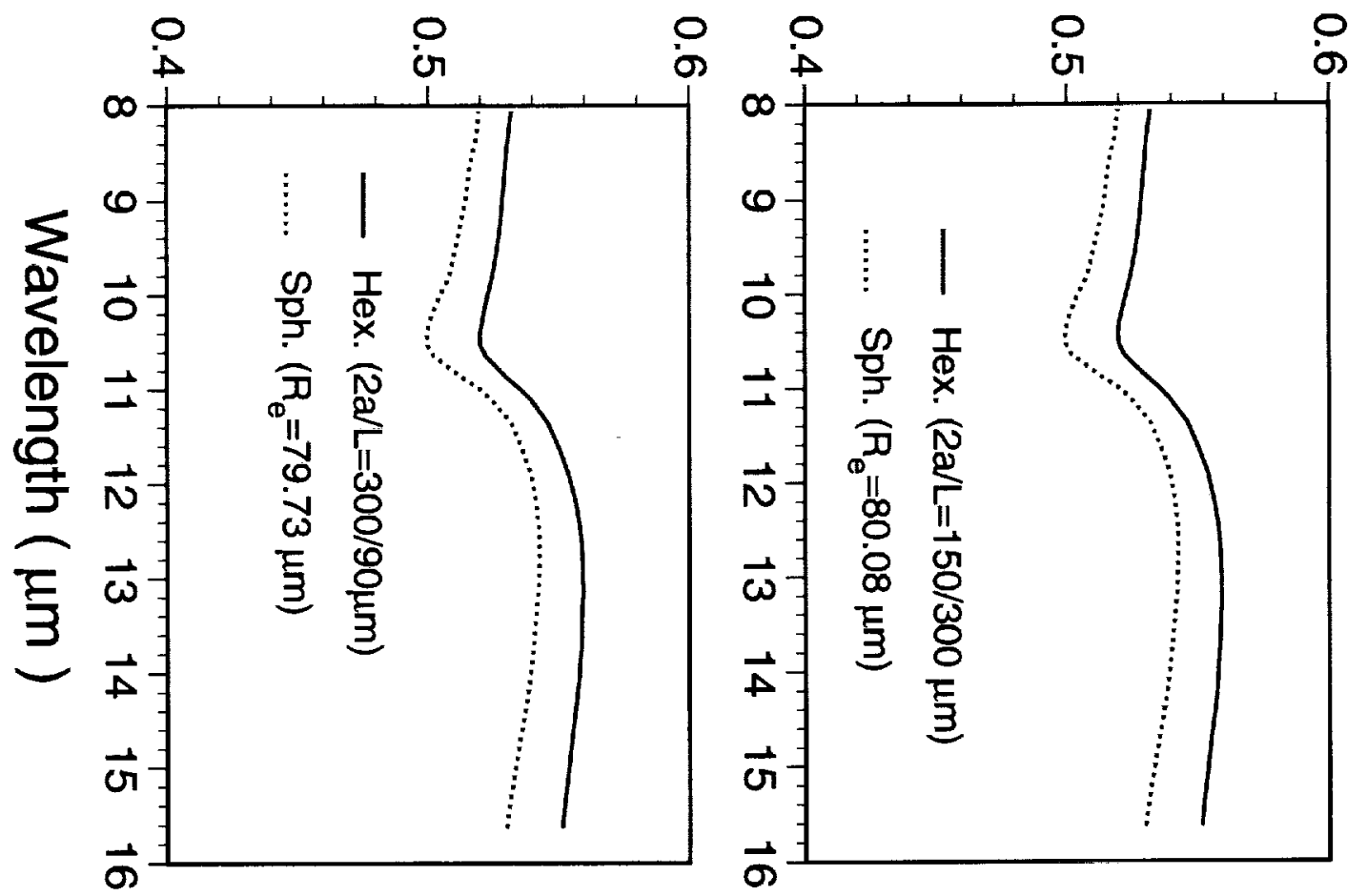


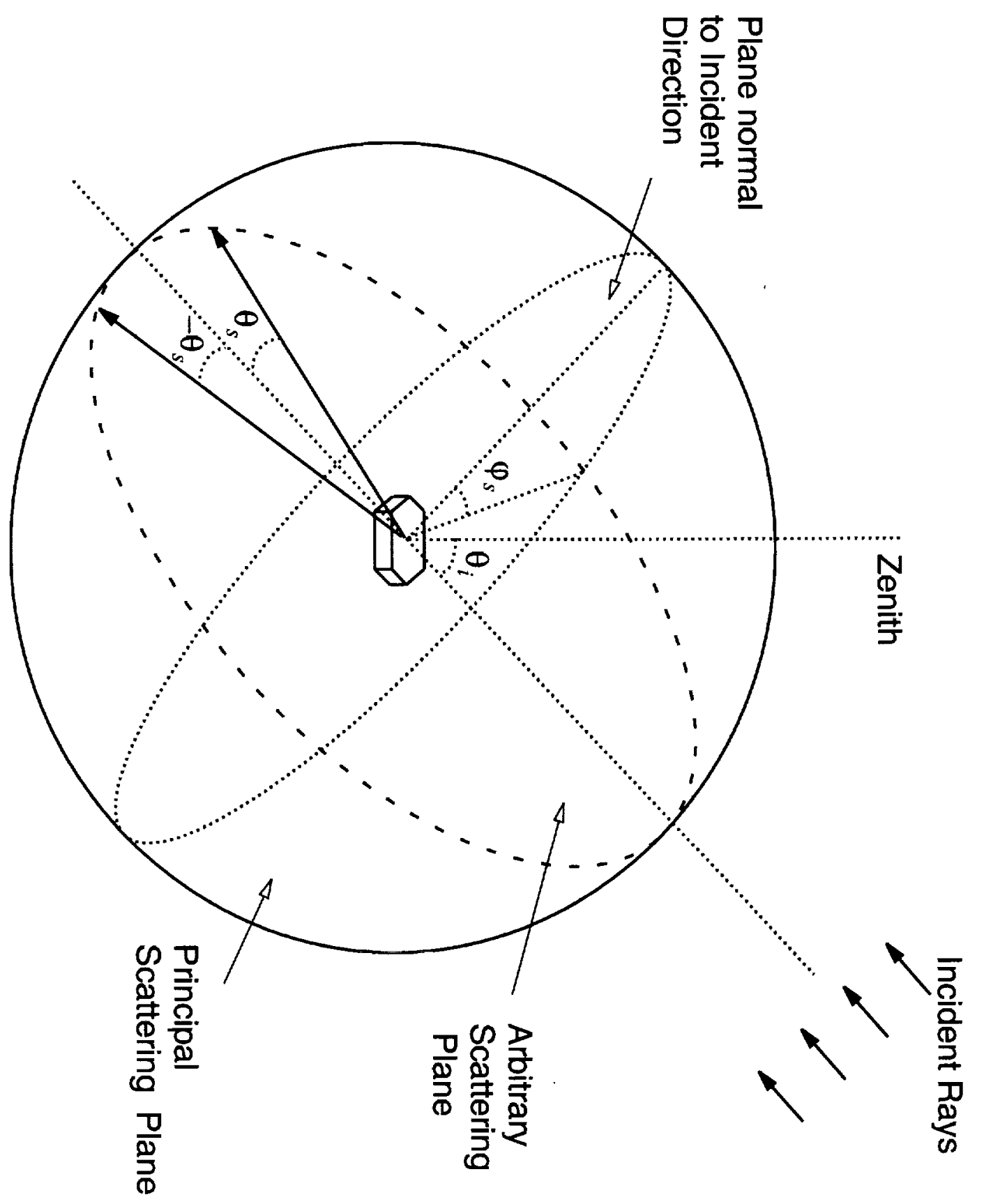

$\stackrel{T}{\underline{0}}$ 


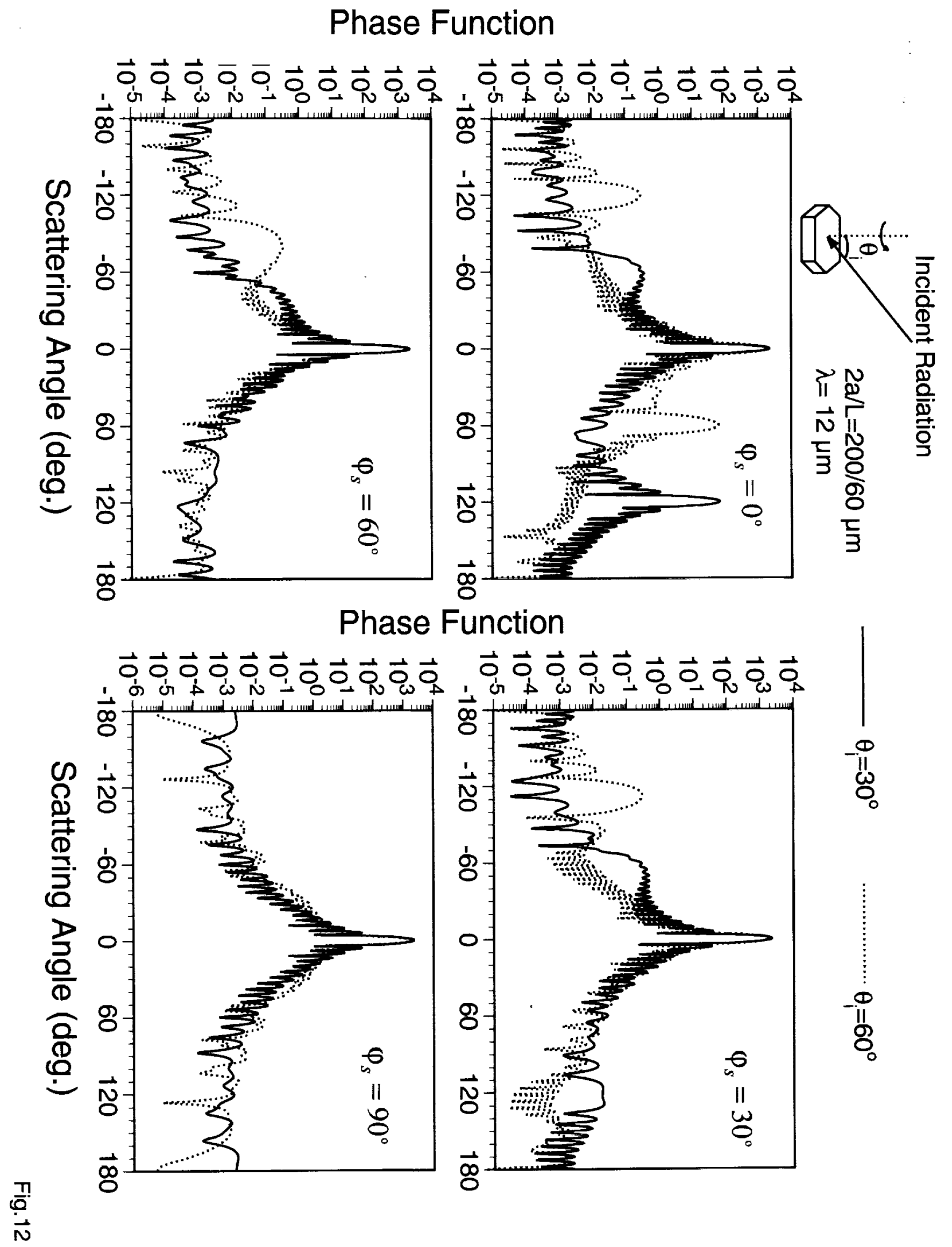


Phase Function
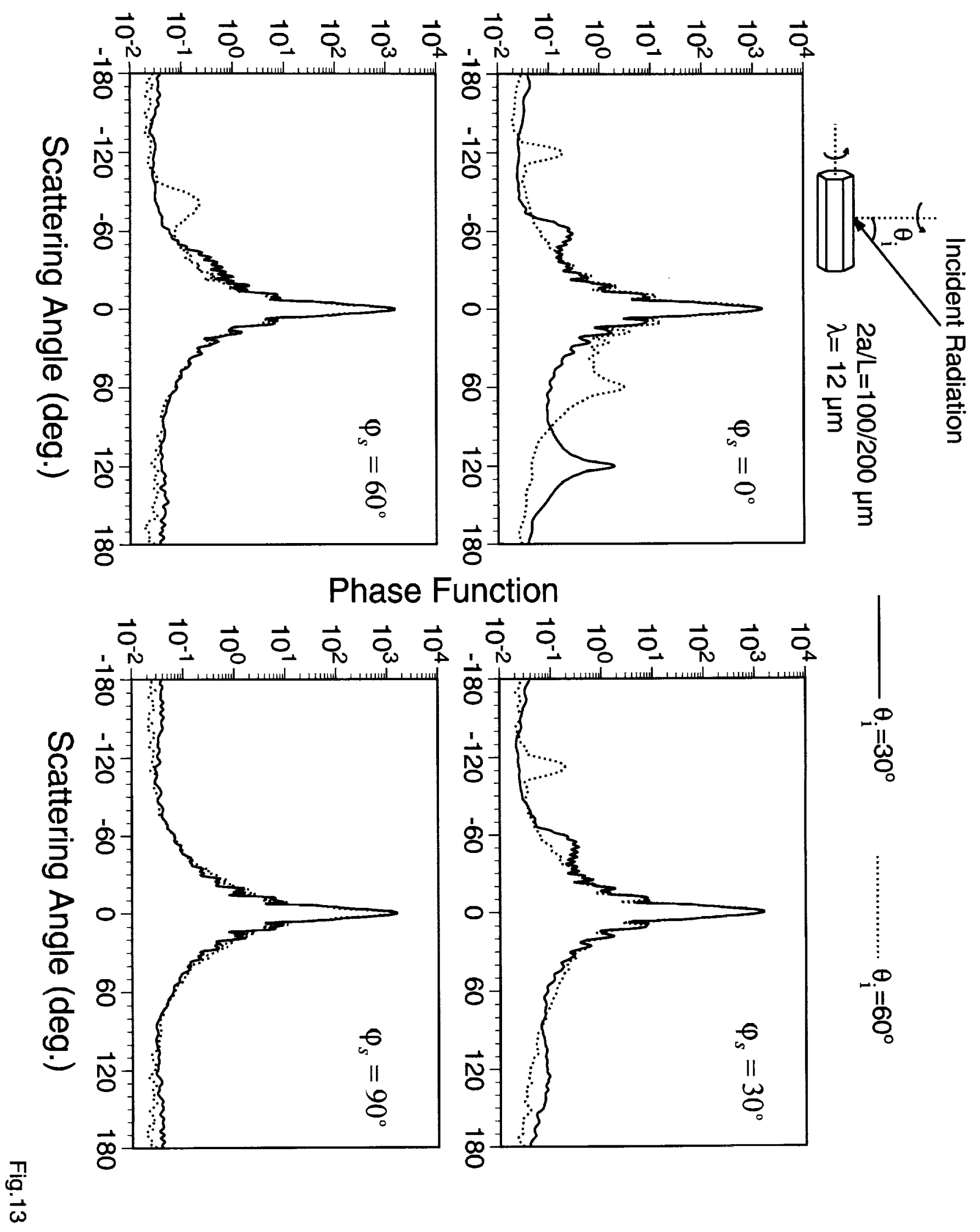

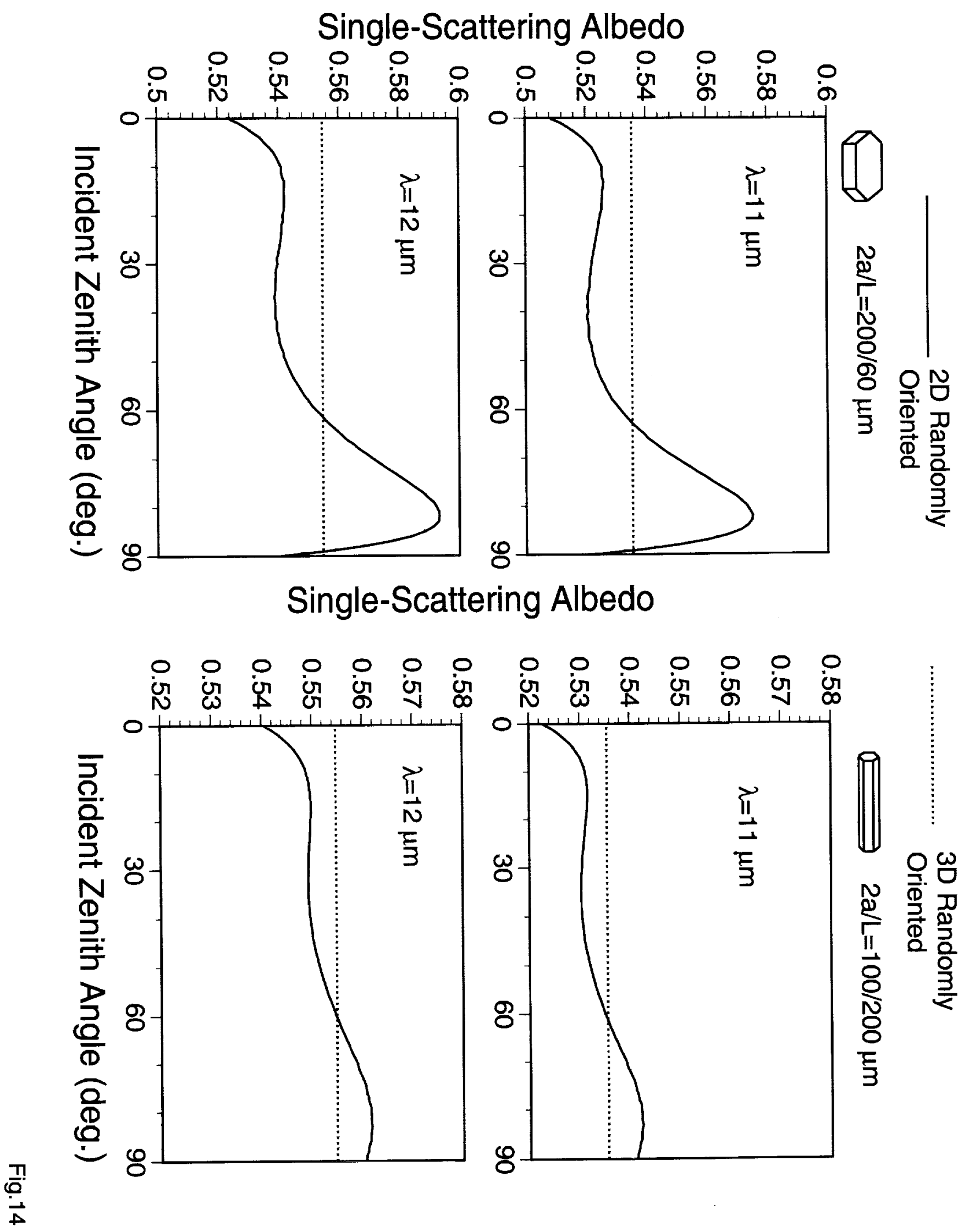